\title{
ESTRATEGIAS DE LEGITIMACIÓN PARA UN ESPACIO DISPUTADO. LA INSERCIÓN DEL INSTITUTO DE PSIQUIATRÍA DE ROSARIO EN LAS REDES Y AGENDA CIENTÍFICA DISCIPLINARES (ARGENTINA, 1929-1944)
}

\author{
José Ignacio Allevi \\ Conicet/ IHUCSO-UNL / UNR, Argentina \\ joseignacio.allevi@gmail.com \\ ORCID iD: https://orcid.org/0000-0003-4819-1398
}

Recibido: 25 enero 2017; Aprobado: 15 enero 2018.

Cómo citar este artículo/Citation: Allevi, José Ignacio (2018), “Estrategias de legitimación para un espacio disputado. La inserción del Instituto de Psiquiatría de Rosario en las redes y agenda científica disciplinares (Argentina, 1929-1944)", Asclepio, 70 (2): p236. https://doi.org/10.3989/asclepio.2018.20

RESUMEN: El trabajo se ocupa del proceso de profesionalización y autonomización disciplinar de la Psiquiatría en la Argentina de entreguerras estudiando una de sus ángulos, referida a la legitimación académica, inserción y posicionamiento en un campo internacional de debates. Para ello toma el caso de la ciudad de Rosario, con la creación del Instituto de Psiquiatría de la Universidad Nacional del Litoral. Éste emergió como resultado de una disputa disciplinar entre neurología y psiquiatría -en su vertiente de higiene mental-, suscitada con la creación de la Facultad de Ciencias Médicas local en 1920. Partiendo de los estudios sociales de la ciencia y la historia psi, se reconstruyen y analizan las estrategias desplegadas por los miembros del Instituto para legitimar y consolidar su disciplina tanto al interior del campo médico rosarino como del campo psiquiátrico en conformación a nivel nacional, regional e internacional, que oscilaron entre la participación en distintos eventos científicos, el sostenimiento de sociabilidades científicas de alcance nacional y latinoamericano y la generación de redes a partir del canje de revistas con distintas latitudes.

PALABRAS CLAVE: profesionalización; campo psiquiátrico; sociabilidad científica; Rosario.

\section{LEGITIMATION STRATEGIES FOR A CONTESTED SPACE. THE INSERTION OF THE INSTITUTE OF PSYCHIA- TRY OF ROSARIO IN DISCIPLINARY NETWORKS AND SCIENTIFIC AGENDA (ARGENTINA, 1929-1944)}

ABSTRACT: The paper deals with the professionalisation and disciplinary autonomy process of Psychiatry in Argentina between the interwar period, studying one of its angles, referred to the academic legitimacy, its entrance and positioning in an international field of debates. For this, it takes the case of the city of Rosario, with the creation of the Institute of Psychiatry of the National Coastal University. It emerged as a result of a disciplinary dispute between neurology and psychiatry -in the orientation of mental hygienearoused with the creation of the local Faculty of Medical Sciences in 1920. Located in the field of social studies of science and psy history, we reconstruct and analyze the strategies deployed by members of the Institute to legitimize and consolidate their discipline, both within the Rosario's medical field and the psychiatric field in formation at a national, regional and international level, wich range from participation in different scientific events, the sustentance of scientific sociabilities of national and Latin American scope, and the network generation from the journals exchange with different latitudes.

KEY WORDS: profesionalisation; psychiatric field; scientific sociabilities; Rosario. 


\section{I - INTRODUCCIÓN}

En junio de 1918, la Reforma Universitaria suscitada en la ciudad argentina de Córdoba convulsionó las bases que estructuraban la organización de la enseñanza superior en Argentina y América Latina (Buchbinder, 2008). Dos años más tarde, en la ciudad de Rosario se institucionalizaba una iniciativa que llevaba casi una década de esfuerzos infructuosos: la creación de su Facultad de Ciencias Médicas (Fernández, 2014), en el marco de la constitución de la reciente Universidad Nacional del Litoral al calor de los eventos reformistas cordobeses (Buchbinder, 2005). Este acontecimiento, si bien abría una serie de posibilidades y recursos de formación académica en una ciudad con una tradición considerable de presencia médica en el espacio público (Prieto, 1996; Pascual, 2017), también nacionalizaba un proyecto educativo concebido localmente, lo que abrió posibilidades al ingreso de nuevos profesionales y lógicas. Por otro lado, esta "novedad" universitaria diferenciaba a la ciudad de otros casos como Buenos Aires, La Plata o Córdoba, que ya contaban con una tradición en este sentido. Rosario abría nuevas posibilidades al desarrollo de corrientes médicas con importante presencia y figuras de peso en la ciudad, como la clínica médica con Clemente Álvarez - la neurología con Teodoro Fracassi, un destacado médico social y políticamente que contaba con el primer espacio de atención de afecciones mentales de la ciudad desde 1914, su "Neuropático". Pero también habilitaba nuevas tendencias. En 1920 un psiquiatra italiano formado con Sante de Sanctis -Lanfranco Ciampi- se instalaba en la Capital Federal, para ser convocado desde dicha unidad académica para radicarse en la ciudad fenicia y desplegar su especialidad, la psiquiatría infantil ${ }^{1}$.

En esta dirección, el Poder Ejecutivo Nacional designó al alienista Antonio Agudo Ávila como delegado organizador de esta casa de altos estudios, ${ }^{2}$ quien introdujo en la currícula de la carrera de medicina una amplia formación psi diferenciada de la tradición local de orientación neurológica (Gentile, 2003), incluyendo tres cátedras especializadas: Psiquiatría de Adultos -a cargo de Gonzalo Bosch-, Neuro Psiquiatría Infantil -a cargo de Ciampi- y Psicología Experimental -dictada por José Alberti-, a lo cual agregaba una Escuela de Niños Retardados desde 1922, anexa a la cátedra de Ciampi, y un Hospital de Alienados, materializado en 1927. Dos años luego se creó el Instituto de Psiquiatría a partir de un proyecto presentado por Raimundo Bosch -docente de Medicina Legal cercano a los psiquiatras-, tras una intensa discusión en los órganos de gobierno de la Universidad donde la corriente neurológica de Fracassi disputó su hegemonía. En efecto, a pesar de que éste neurólogo ya contaba "formalmente" con un Instituto de Psico Neuro Patología creado en 1924, el contexto de conflictividad universitaria de 1928 -que traducía las disputas faccionales internas del partido político gobernante, la Unión Cívica Radical- derivó en la intervención de la Universidad, resolviéndose el conflicto con la disolución por decreto del Instituto de Fracassi y la creación del nuevo Instituto, concreción que dio lugar a un completo dispositivo que nucleaba instancias de docencia, investigación y clínica, y que fue posible tanto por el arribo de nuevos profesionales, y con ellos de nuevas tendencias intelectuales, así como también por un entramado vincular en distintos niveles de la política nacional, provincial y local (Allevi, 2017).

Este trabajo es parte de una investigación que se pregunta por la construcción de un espacio de ciencia legítimo para la Psiquiatría en la ciudad de Rosario, como localización de un proceso trasnacional de profesionalización de la psiquiatría durante la entreguerras, así como de constitución de un campo psiquiátrico en Argentina. En las últimas décadas se configuraron algunas líneas de trabajo en historia de la psiquiatría: los intentos por liberarse de la genealogía antipsiquiátrica, un enfoque interdisciplinar enmarcado en los estudios sociales de la ciencia, aquellos centrados en el material turn que aborda la atención psiquiátrica -incluyendo registros de pacientes para observar la dinámica práctica entre saberes y sujetos-, la llamada historia cultural de la psiquiatría, o bien la problematización de la recepción, circulación y praxis de saberes psiquiátricos (Hess y Majeurs, 2011; Huertas, 2001, 2012, García, 2012).

Por otra parte, en lo referido a los procesos de profesionalización de la atención en salud, encontramos en el caso de Argentina abordajes específicos que rastrean desde fines del siglo XIX y las primeras décadas del XX estos procesos para la medicina académica en Capital Federal (González Leandri, 1999; 2012), las tensiones e hibridaciones entre artes de curar indígenas, populares y diplomados en el área pampeana ( $\mathrm{Di}$ Liscia, 2003) o bien la especialización y aceptación pública de algunas disciplinas, como la tisiología (Armus, 2007; Carbonetti, 2003) o las profesiones "auxiliares", como enfermería (Ramacciotti y Valobra, 2010). En el área específica de la psiquiatría, indagaciones de este tipo son recientes en Argentina, y se ubican temporalmente en el período de la entreguerras. Algunos han problematizado de manera sugerente el abordaje in- 
telectual, con los estudios de la ciencia, y así complejizar la circulación trasnacional de saberes psi mediatizados por cosmovisiones más amplias y estructuras político-partidarias, en el caso del Partido Comunista en Argentina y la psicología soviética (García, 2016). Otros trabajos, por su parte, abordaron los debates intelectuales en su actualización práctica al trabajar con producciones teóricas junto al campo de su aplicación clínica en el caso Hospital Esteves de la provincia de Buenos Aires (Golcman, 2015; 2017).

Cierto es que, a diferencia de otros espacios iberoamericanos, el desarrollo de la historia psi en Argentina sostuvo un perfil transdiciplinar que permitió abordar entrecruzadamente psiquiatría, psicología o psicoanálisis, y no de manera separada como en otros casos. En la bibliografía disponible ha primado un enfoque intelectual que adquirió distintos matices y niveles de complejidad. Algunos estudios se preocuparon por los desarrollos tempranos de la disciplina psicológica, dando cuenta de la circulación de teorías y conceptos en la enseñanza impartida en la Universidad de Buenos Aires -entre ellos el Degeneracionismo o el "Darwinismo Social"-, así como sus aplicaciones en áreas disciplinares tan diversas como el Higienismo, la Educación o la Criminología, entre fines del siglo XIX y las primeras dos décadas del XX (Vezzetti, 1983; Talak, 2007, 2010). Con un importante aporte a la circulación de ideas y debates en la ciudad de Buenos Aires y algunas de las ciudades centrales del interior del país, estos trabajos han considerado los "dilemas" de la recepción, problematizando las reconceptualizaciones locales de los saberes psi europeos. Otras pesquisas abordaron localmente distintas facetas de la historia psi en su amplia acepción -psiquiatría, psicología, psicoanálisis- para los casos de Rosario (Gentile, 2003) o Córdoba (Ferrari, 2016). Pueden señalarse, sin embargo, algunas vacancias que la reducción de la escala de análisis permite vislumbrar, en particular el plano de la gestión institucional de los recursos para poder desarrollar un espacio de ciencia y producción, así como las estrategias desplegadas por los agentes para insertar concretamente sus saberes en determinadas esferas, como la estatal. Partiendo del investigaciones que pensaron este punto para el caso español (Huertas, 2008, 2002), nuestro estudio pretende abonar a esta vacancia que resulta tan relevante como la intelectual siendo, como sostenemos, su condición de posibilidad.

Una copiosa bibliografía, por otra parte, se dedicó en el caso argentino a la creación de institutos de investigación como los ámbitos por excelencia que faci- litan procesos de autonomización y profesionalización disciplinar para áreas tan diversas como la medicina (Buschini, 2013; Romero, 2016), la astronomía (Rieznik, 2009) o la matemática (Pacheco, 2010). Si por un lado estas pesquisas señalaron oportunamente la importancia de las redes en la recepción de ideas y prácticas científicas, no dan cuenta, en cambio, de los vínculos sostenidos por los actores que investigan con el mundo no científico, y principalmente con el Estado. Por otro, la mayoría de ellos se centra en el período del primer peronismo como momento de inicio de la promoción estatal de la ciencia, cuando un rastreo sobre el trabajo de lobby de los científicos durante la entreguerras como gestores y "publicistas" de su trabajo da cuenta de las posibilidades que encontraron, así como de los antecedentes que sentaban. A este respecto cabe señalar que existen investigaciones que abordaron agudamente la conformación de las redes internacionales entre científicos locales adeptos a la eugenesia (Miranda, 2013), así como las vinculadas al problema de la cultura física, en un contexto de circulación trasnacional de debates en torno a la medicina deportiva, y su emergencia como campo disciplinar (Reggiani, 2016).

En este trabajo abordaremos una faceta de dicho proceso, referido a las estrategias de los miembros del Instituto para legitimar y consolidar su disciplina científica tanto al interior del campo médico rosarino como del campo psiquiátrico en conformación a nivel nacional/regional e internacional, para lo cual consideraremos la participación en distintos eventos científicos, la construcción de sociabilidades científicas de alcance nacional y latinoamericano y la construcción de redes a partir del canje de revistas con distintas latitudes

Frente al reduccionismo de algunos estudios de redes en su faz estrictamente descriptiva de axiomas, rescatamos la necesidad de recurrir no sólo a los contextos que las vuelven posibles, sino $-y$ fundamentalmente- al problema de las relaciones diádicas que las constituyen: el contenido de los vínculos (Bidart y Cacciuttollo, 2009) y las mediaciones que las vuelven posibles (Grossetti, 2009). Las revistas, por su parte, son pensadas aquí como soportes de sociabilidad al interior del campo intelectual (Dosse, 2006).

En sus apartados segundo y tercero, el artículo aborda el período inicial del Instituto de Psiquiatría de Rosario, para analizar la dinámica que tuvo la construcción de redes en los planos de sociabilidad científica y participación en eventos, así como el trazado de redes internacionales a partir del canje de su Boletín. En la cuarta sección analizaremos la "segunda época" del organis- 
mo, para mostrar los cambios significativos operados en su funcionamiento y su autonomización, pero sobre todo en la posición que alcanzó en un campo disciplinar psiquiátrico progresivamente delimitado.

\section{II - LA PRIMERA ETAPA DEL INSTITUTO (1929-1934): SOCIABILIDAD Y DIFUSIÓN DE LA CIENCIA}

Una vez creado el Instituto, los psiquiatras de Rosario emprendieron un conjunto de estrategias de vinculación científica para posicionarse en el campo psiquiátrico en conformación en tres niveles: al interior del campo médico local-hegemonizado por el Círculo Médico de la ciudad-; a nivel regional/nacional, y en el plano internacional. El conjunto de esfuerzos -participación en eventos científicos, conferencias, cursos, y generación de redes- se daba en su búsqueda de un estatuto de cientificidad para su alcanzar legitimidad y reconocimiento, en medio de su pugna por autonomizarse en un área disciplinar específica en el cuidado de la salud. En esta dirección, tanto a nivel colectivo como individual, los galenos operaron en una doble dirección: ubicarse en distintos espacios de reconocimiento, intercambio y discusión científica, por un lado; y tramando de redes intelectuales nacionales e internacionales a partir del canje de su publicación oficial con otras agencias académicas, por otro.

En años iniciales del Instituto de Psiquiatría, la presencia en eventos académicos se resume en dos nombres: Lanfranco Ciampi y Gonzalo Bosch, quien desde el inicio de la década del treinta se tornó una figura clave en el campo al acceder a la dirección del Hospital de las Mercedes de Capital Federal -espacio central en la hegemonía psiquiátrica-, además de presidir la Sociedad de Neurología y Psiquiatría de Buenos Aires, la Liga Argentina de Higiene Mental, y numerosas revistas y espacios académicos.

Un evento en particular permitió la visibilización y vinculación de los psiquiatras en la etapa en que se estaban discutiendo los proyectos de creación del Instituto en los órganos de gobierno de la Universidad del Litoral: la Primera Conferencia Latino-Americana de Neurología, Psiquiatría y Medicina Legal llevada a cabo en Buenos Aires durante noviembre de 1928. Presidida por Arturo Ameghino ${ }^{3}$-quien había trabajado junto a Ciampi-, su realización fue posible por una intensa labor de sociabilidad académica que generó conflictos disciplinares al interior de la Sociedad de Neurología y Psiquiatría dependiente de la Asociación Médica Argentina, su institución convocante ${ }^{4}$. Este espacio es central para pensar los vínculos previos a la constitución del Instituto de Psiquiatría de Rosario, así como su deriva en nuevos espacios de intercambio, aunados en sus orígenes por haber surgido de escisiones en grupos que priorizaban el desarrollo de la Neurología en desmérito de la Psiquiatría ${ }^{5}$. Su presidente planteaba esta disputa al inaugurar la Conferencia:

"Es de ese modo que la propia ciencia médica se asocia al prejuicio social para conspirar sin quererlo contra la Psiquiatría; y es por ello que el psiquiatra, peregrino en las clínicas según el público astuto, y en realidad paria en el seno de la profesión, cede a su impotencia, déjase invadir por la quietud, y conspira él también con su silencio contra los intereses generales." (Ameghino, 1929a, p. 40-41)

La comisión organizadora y directiva de la Conferencia reunía una serie de personalidades locales e internacionales que conformaron de allí en más una red actualizada en distintos eventos a lo largo de la década del treinta. Gonzalo Bosch era vocal de dicha comisión, mientras que los delegados por la Facultad de Medicina de Rosario eran Teodoro Fracassi, Ciampi y Raimundo Bosch, aunque Ciampi figuraba, además, como representante del Hospital de Alienados de Rosario. Entre el resto de los delegados locales e internacionales con quienes luego se sostendrán vínculos, cabe mencionar a Nerio Rojas, José Belbey, Osvaldo Loudet, Gregorio Bermann, Emilio Catalán, Herminio Valdizán, Henrique Roxo, Antonio C. Pacheco Silva, Ernani Lopes ${ }^{6}$.

En lo que refiere a la participación de los psiquiatras rosarinos y sus colegas porteños, Ciampi, Raimundo y Gonzalo Bosch expusieron varios trabajos durante la segunda sesión del evento. Allí, el psiquiatra italiano sostuvo un intenso diálogo con la ponencia de Ernani Lopes, quien presentó "De las psicosis infantiles y especialmente de la parálisis general infantil" (Ameghino, 1929a, p. 442-469). Éste último, miembro de la Liga Brasilera de Higiene Mental, contribuiría a la designación de Ciampi y Bosch como miembros correspondientes de dicha entidad -véase infra-. Ahora bien, en el mismo momento en que se suscitaba en Rosario la disputa por la creación del Instituto -y en especial por su dirección, donde se dirimía la independencia o subordinación de la psiquiatría a la neurología-, llama la atención las cinco presentaciones que Teodoro Fracassi realizó sólo o en coautoría en las distintas sesiones (Ameghino, 1929a, 1929b). Vemos, así, que en dicho contexto este tipo de eventos resultaba una oportunidad tanto para intercambiar y generar lazos como para medir fuerzas entre los grupos en pugna por la hegemonía de la enseñanza y atención psi en Rosario.

Estas vinculaciones mencionadas se hicieron patentes una vez creado el Instituto, en 1929. A princi- 
pios de dicho año, Ciampi fue invitado con Cayetano Viale ${ }^{7}$ por Gregorio Bermann ${ }^{8}$ y el Círculo Médico de Córdoba para dictar dos conferencias en la ciudad mediterráne, además de participar en la cátedra de Medicina Legal de este último médico. ${ }^{9}$ La relación con Bermann es un punto interesante en la vida del Instituto de Rosario puesto que, por una parte sostuvo un vínculo fluido con alguno de sus miembros -en particular Ciampi-, mientras que, por otra, en 1933 el psiquiatra cordobés concursó por la cátedra de Clínica Psiquiátrica, ante lo cual prevalecieron los intereses locales y se excluyó su candidatura por su filiación política ${ }^{10}$. Su compromiso con el antifascismo y su participación en la Guerra Civil Española, empero, fueron un punto de unión con la política de la Universidad del Litoral de acoger profesionales ibéricos exiliados (De Marco, 2015; Farías, 2013).

En el mismo año, la articulación de vínculos entre Rosario y Buenos Aires se consolidó con la creación de la Liga Argentina de Higiene Mental -en adelante LAHM-, motorizada por Gonzalo Bosch como desprendimiento de la Sociedad de Neurología y Psiquiatría de Buenos Aires mencionada previamente, que continúo funcionando ${ }^{11}$. Ésta resultó central en varios sentidos. En primer lugar, convocaba un nutrido grupo de especialistas, favoreciendo el intercambio científico y académico, además de facilitar la concreción de proyectos de intervención social a partir del capital social o político de uno o varios de sus miembros. Entre éstos, encontramos a Luis Estévez Balado, Fernando Gorriti, Ramón B. Silva, Arturo Mó y Antonio Martínez en los cargos de gestión, mientras que sus vocales titulares fueron Ciampi, Arturo Ameghino, Juan M. Obarrio, Julio C. Nogués, Juan C. Montanare, Julio Oliveira Estévez, Roque Orlando, Alberto Zwank y José Belbey. Mientras que en los suplentes vemos a Nerio Rojas, Santiago Balestra, Osvaldo Loudet, Eusebio Albina y Julio C. Hanón. Muchos de estos personajes generaron luego vínculos académicos a partir del intercambio de sus publicaciones. En segundo término, y de manera similar a otras asociaciones médicas organizadas según el mismo esquema, la $L A H M$ desarrolló su programa de intervenciones en Buenos Aires (Talak, 2005), y promovió una serie de "comités regionales" en las ciudades de Rosario, Santa Fe, Mendoza y Córdoba. Por último, concretó un espacio académico -y simbólico- para una demanda de larga data entre los especialistas en medicina mental: la institucionalización de la corriente de la Higiene Mental, en tanto propuesta teórica que -en Argentina- permitió a la Psiquiatría diferenciarse de la Neurología y del campo de la Medicina en general.
En julio de 1930 se llevó adelante en Río de Janeiro y San Pablo la Segunda Conferencia Latino-Americana de Neurología, Psiquiatría y Medicina Legal, en la cual nuevamente participaron los docentes de la Facultad de Medicina de Rosario, pero bajo un estatuto distinto. En el marco de la intervención política de la Facultad de Medicina -que facilitó la creación del Instituto de Psiquiatría-, Ciampi y Gonzalo Bosch fueron designados delegados oficiales por la casa de altos estudios, el Estado Nacional y provincial de Santa Fe, una cuestión interesante para considerar la posición fortalecida que ocupaban en ese momento ${ }^{12}$. Su presencia en la Conferencia, además, fue significativa de manera bifronte. Por un lado, allí fueron designados representantes argentinos de la Liga Brasilera de Higiene Mental, un acto simbólico y revelador de la intención de fortalecer institucionalmente ciertos vínculos, a partir de los cuales instituciones brasileras devinieron una constante en la vida relacional del Instituto de Psiquiatría. Por otro, su participación académica en las jornadas incluyó un total de once trabajos que incorporaban a miembros del Instituto, como Carlos Crespo, José Cid, Antonio Foz, Eleogardo Troilo, José Alberti, Arturo Mó, junto a las colaboraciones de Raimundo Bosch. Las temáticas de sus presentaciones respondieron a dos "criterios": aquellos estrictamente médicos -donde se destaca la "Clasificación de las enfermedades mentales" propuesta por Ciampi y Bosch- y otros destinados a publicitar la organización de los estudios psiquiátricos en la Facultad fenicia, en particular la incorporación de la Psicología Experimental y la especialización infantil lo cual despertó "la unánime aprobación por lo que ha ya realizado nuestra Facultad y por lo que tenta actualmente realizar a este respecto la Facultad de Medicina de París." ${ }^{13}$.

Un año más tarde, y bajo la presidencia de Gonzalo Bosch, la Sociedad de Neurología y Psiquiatría de Buenos Aires -en la cual aún participaban médicos de la LAHM- celebró sus sesiones extraordinarias en el Círculo Médico Rosario y el Instituto de Psiquiatría, lo que propició un fluido intercambio entre profesionales de Rosario, Capital Federal y Córdoba. Pero además, dichas jornadas se desarrollaron en ámbitos que vinculaban a Fracassi y al grupo de los higienistas mentales, y ello se evidenció al comparar el número de ponencias presentadas por ambos grupos. De hecho, Fracassi realizó tres presentaciones individuales y diez en co-autoria con distintos miembros de su grupo -Leonardo Parachú, Fernando Ruiz, Alfonso Quaranta, Demetrio García-, mientras que Ciampi presentó un solo trabajo individual y dos en conjunto. Gonzalo Bosch, por su parte, participó en cinco ponencias con 
otros colegas. Otros miembros del Instituto también expusieron sus investigaciones de manera individual, como fue el caso de José M. Cid y Arturo Bruno -jefes de los laboratorios de Histopatología del sistema nervioso y de Bioquímica, respectivamente-, situación que no se daba con los neurólogos. Otros miembros de la Sociedad que participaron fueron Braulio Moyano, Christofredo Jakob, Roque Orlando, Luis Estévez Baldao, Gregorio Bermann, Enrique Mouchet, José Alberti, Enrique Món ${ }^{14}$

A estas sesiones extraordinarias debemos agregar la inclusión de Ciampi y Raimundo Bosch en el comité ejecutivo del Congreso Nacional de Neurología, Psiquiatría y Medicina legal, a realizarse en octubre de 1931, organizado por la misma Sociedad en su sede de Buenos Aires, ${ }^{15}$ además de la participación del psiquiatra italiano junto a José Alberti en la Sociedad de Psicología de Buenos Aires, ${ }^{16}$ con un comunicación sobre "El reflejo psicogalvánico" ${ }^{17}$. Es de destacar que hayan sido convocados a participar en este espacio, puesto que el único lazo concreto que los vinculaba a este último eran las figuras de José Alberti -ya en retirada del Instituto-, y la de Arturo Ameghino.

En otro orden, en la primera etapa del Instituto el área más destacada en su difusión e "internacionalización" fue la de psiquiatría infantil promovida por Lanfranco Ciampi, donde los mayores elogios provenían del viejo continente, un ámbito privilegiado donde el psiquiatra italiano dio a conocer su experiencia, a lo cual deben sumarse sus reportes epistolares regulares sobre sus logros a Sante De Sanctis ${ }^{18}$.

Con el impulso recibido a su especialidad en la Facultad de Medicina de Rosario, vemos que en 1929 la Revista Infanzia Anormale se pronunciaba sobre la creación de la especialidad de Neuro Psiquiatría Infantil, donde reconoce al organizador de la Facultad, Agudo Ávila como el principal propulsor de un espacio para la autonomización disciplinar ${ }^{19}$. En 1932, Henri Claude envió personalmente la última edición de su tratado de Neuropatología al Instituto, el cual se encargan de reseñar; $y$, por otra parte, en ese mismo año Pierre Janet visitó las instalaciones de la Facultad de Medicina ${ }^{20}$. Al conocer la Escuela para niños retardados, afirmó "con toda mi admiración por la pequeña Clínica Psicopatológica; es el sueño de toda mi vida que veo aquí realizado."21.

A finales de dicho año, se celebraron en Montevideo las Jornadas Rioplatenses de Neuropsiquiatría, gestadas entre Gonzalo Bosch y su par uruguayo Antonio Sicco, que contaron con la par- ticipación de un nutrido grupo de la Sociedad de Neurología y Psiquiatría, que Bosch aún dirigía. Allí los integrantes del Instituto participaron con 8 trabajos, y Ciampi recibió un reconocimiento oficial por parte del Consejo Nacional de Enseñanza Primaria y Normal de Uruguay, a partir de la influencia que uno de sus miembros ejerció, tras haber visitado apenas unos años atrás las instalaciones del dispositivo rosarino:

\footnotetext{
"Señor Dr. Lanfranco Ciampi. - El Consejo Nacional de Enseñanza Primaria y Normal, en conocimiento de que el Sr. Profesor se hallaba en nuestra ciudad, y considerando sus altos merecimientos científicos y la cordial gentileza con que nos acogió en ocasión de las visitas efectuadas a los establecimientos de su brillante dirección, resolvió en la sesión del día 28 , designar una Comisión integrada por su presidente Dr. Santín C. Rossi, el Consejero Sr. Emilio Verdesio y el Inspector Técnico Sr. Luis Pecantet, para trasmitirle el saludo del Consejo y hacerle lo más grata su estadía entre nosotros..." 22
}

Hacia 1933 se constituyó en la ciudad de Buenos Aires otro espacio relevante de sociabilidad científica con la Sociedad Argentina de Criminología, dirigida por Osvaldo Loudet, Carlos de Arenaza y Antonio Beruti, figuras ya participantes en los espacios anteriores. Con sede en el Instituto de Criminología de la Penitenciaría Nacional -en la Dirección General de Institutos Penales-, la asociación emergía de la Revista de Criminología, Psiquiatría y Medicina Legal -ahora bajo la dirección de Loudet-, y operaba como instancia de intercambio científico y circulación de saberes entre médicos, políticos, abogados y magistrados con eje en problemáticas socio-penales específicas. Allí se insertaron Ciampi, Gonzalo y Raimundo Bosch -como socio corresponsal en Rosario-. Entre sus integrantes, contamos en el ámbito nacional con Arturo Ameghino, José Belbey, Víctor Delfino, Jorge E. Coll, Roberto Ciafardo, Luis Estevez Baldao, Héctor Piñero, Telma Reca, Alejandro Raitzin y Nerio Rojas. Como "miembros correspondientes" aparecían Gregorio Bermann, Hernani Lopes, Emilio Catalan y Carlos Bambarén, mientras que sus miembros honorarios extranjeros incluían a Luis Jiménez de Asúa, Flaminio Favero, Carlos A. Pacheco e Silva (ambos de San Pablo), Afranio Peixoto y Leonido Ribeiro (Río de Janeiro) ${ }^{23}$. En efecto, las redes se fortalecían: el Instituto canjeaba su publicación con los últimos profesionales lusitanos, Ciampi sostenía un vínculo con Coll y Ameghino desde su llegada al país, Reca participaba de la LAHM y hemos revisado ya el vínculo con Bermann. 


\section{III - CIRCULACIÓN E INTERCAMBIO DE REVISTAS: EL BOLETÍN DEL INSTITUTO PSIQUIÁTRICO EN SU “PRI- MERA ÉPOCA"}

A partir de su materialización, en los inicios de 1929 comenzó a publicarse el Boletín del Instituto Psiquiátrico, aparición recibida rápidamente en uno de los principales órganos de la especialidad, la Revista de Criminología, Psiquiatría y Medicina Legal, dirigida en ese momento por Osvaldo Loudet. A partir de la cercanía y los distintos círculos que vinculaban a los miembros del Instituto con la esfera de dicha publicación, vemos que ya en el número 97 y 98 se reseñaban tres artículos de la edición inicial del BIP. ${ }^{24}$ Presidido por Ciampi en tanto director del Instituto y con Eleogardo Troilo como secretario de redacción, su comité de redacción estaba integrado por los docentes miembros, los Jefes de Laboratorio y los médicos agregados al hospital ${ }^{25}$.
Concebido como una publicación trimestral, funcionó de esta manera por tres años, mientras que en 1932 comenzaron los períodos alternos. Ese año se publicó un sólo número para todo el año lectivo; en 1933 se editan dos ejemplares, para luego publicar un solo número que abarcó el periodo de septiembre entre 1933 y 1934 . Aquí se interrumpió su publicación por dos años completos, al coincidir la falta de partidas presupuestarias para su edición con un conflicto suscitado durante la intervención del Hospital por las autoridades universitarias en $1934^{26}$. Este obstáculo, empero, no implicó una merma en los intercambios, por dos razones. Por una parte, las sociabilidades se sostuvieron por otras vías generadas previamente; por otra, el Boletín se había insertado en redes de canje que aguardaban su publicación para sostener el vínculo a través del intercambio, como observamos en las notas que el comité de redacción recibió (Ver imágenes 1 y 2 ) de la Revista Médica Latino Americana y los Archivos Uruguayos de Medicina, Cirugía y Especialidades ${ }^{27}$.

\section{Imagen 1}

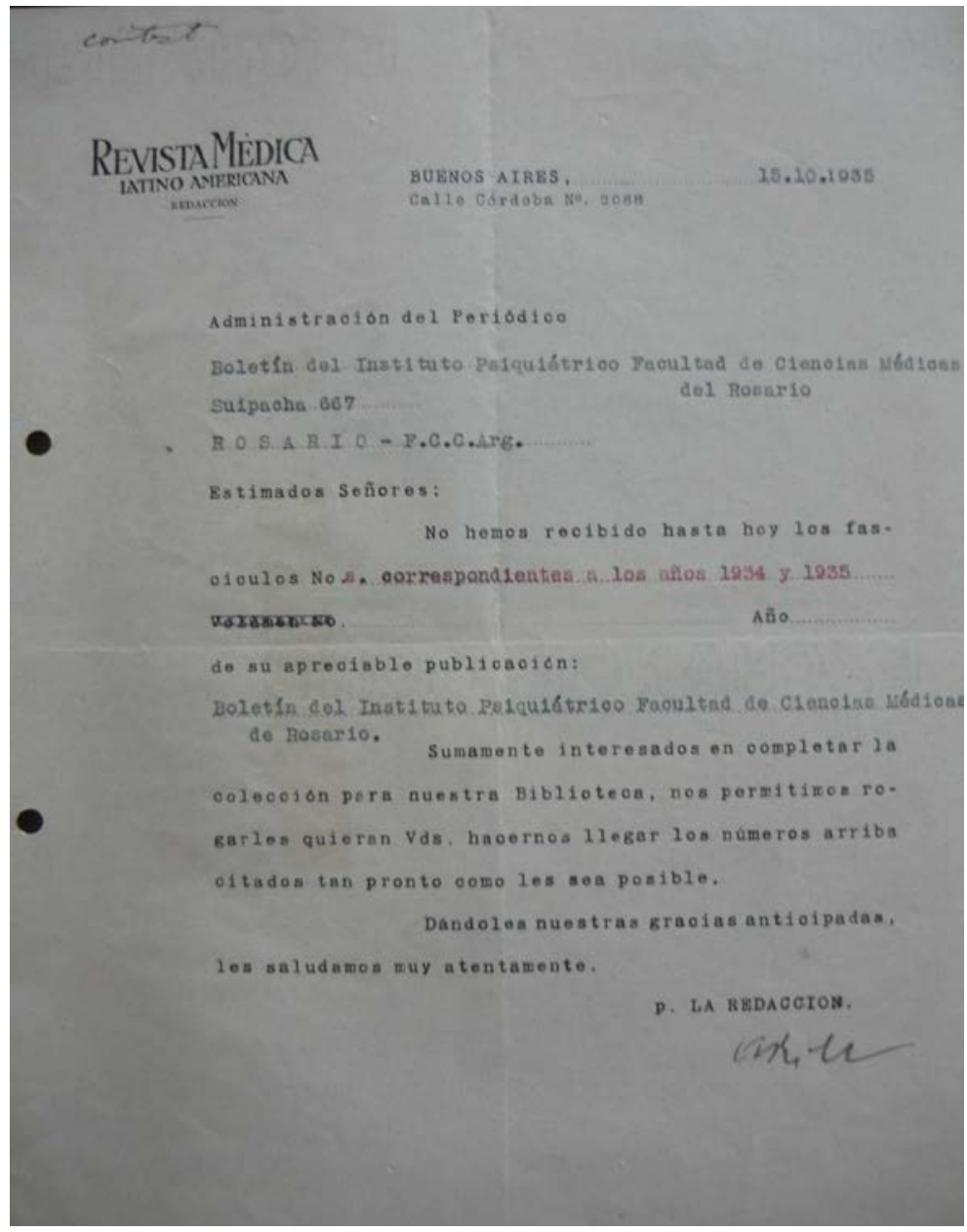


Imagen 2

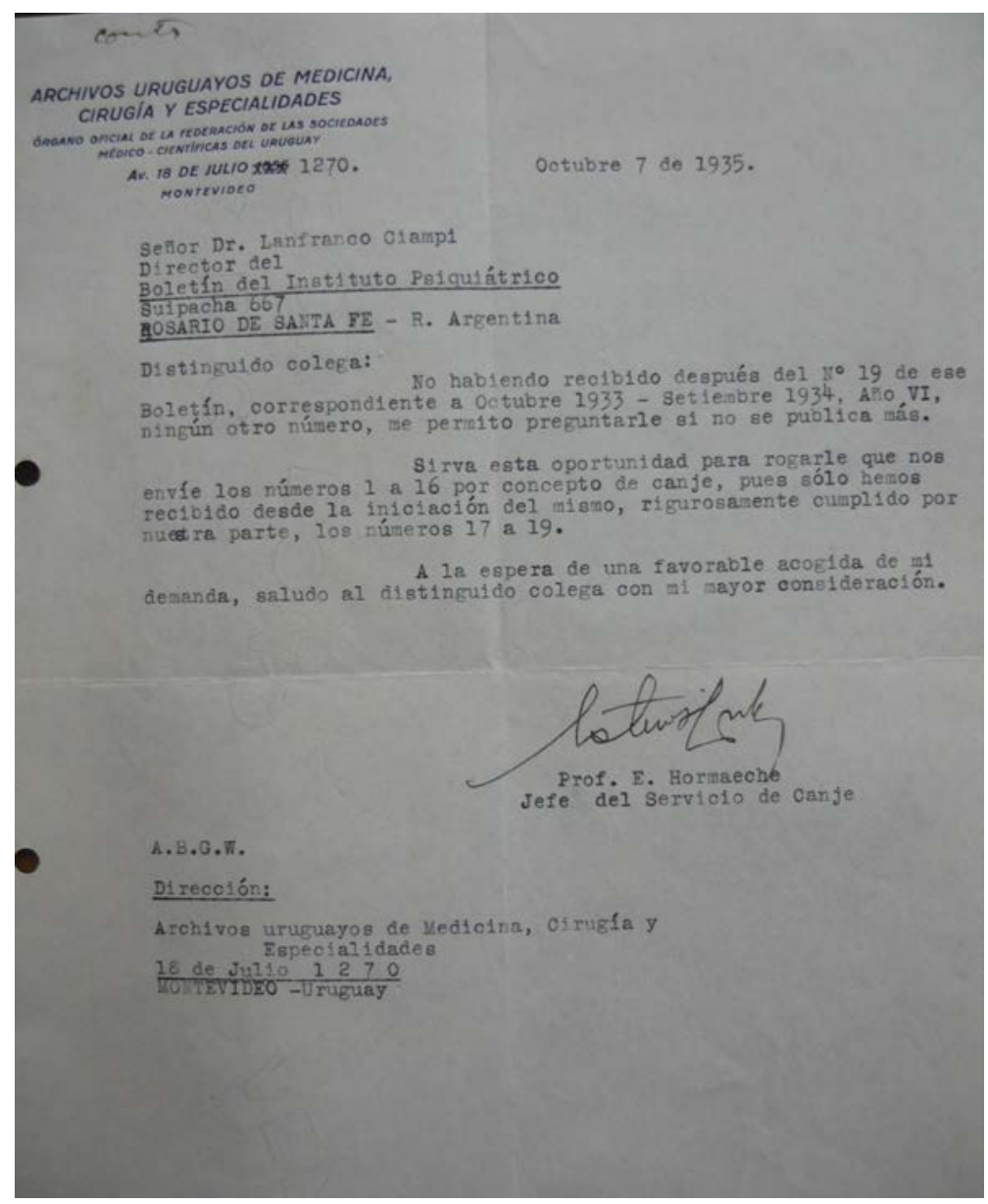

Si consideramos, entonces, el intercambio de revistas a escala internacional, es posible reconocer un claro predominio las producciones italianas durante la primera etapa del Instituto, entre cuyos principales títulos se reseñan numerosas publicaciones de órganos asociados al fascismo. Entendemos que esta primacía responde a la acción de Ciampi como "publicista" de su instituto entre los centros de investigación de su país natal, y en especial con su mentor intelectual, Sante de Sanctis, aunque los vínculos se extendieron por fuera del espacio itálico (Ver tabla № 1 y 2). Como puede verse en el inventario de direcciones disponible en el Instituto, el Boletín se enviaba a destacadas personalidades de la escena psiquiátrica europea, y muchas, como Henri Claude, Èdouard Claparède, Eugen Bleuler, Gregorio Marañón o Emilio Mira y López. ${ }^{28}$ Cabe destacar que, entre los nombres del listado, encontramos un número importante de especialistas en psiquiatría infantil, educación o pre- cursores del movimiento de higiene mental, áreas específicas de Ciampi (Ver tabla no 3).

\section{IV - LA “SEGUNDA ÉPOCA” DEL INSTITUTO (1934-1944)}

El segundo período identificable en la vida del Instituto inicia con dos eventos considerables: por una parte, la suspensión de la edición de su Boletín, en el mismo momento que el grupo de Neurología iniciaba su propia publicación, la Revista Argentina de Neurología y Psiquiatría, dirigida por Fracassi. Por otra, la renuncia de Lanfranco Ciampi a su dirección a finales de $1934^{29}$. Frente a las reticencias de las autoridades universitarias a aceptarla, se le propuso tomar licencia de la dirección del Hospital, hasta que en 1936 finalmente la aceptan, y quien lo reemplazó en dichas funciones, Antonio Foz -titular de Psiquiatría de Adultos con la renuncia de Bosch-, fue quien ocupó definitivamente el $\operatorname{cargo}^{30}$. Por otro lado, a lo largo de esta nueva época, creció de manera considerable el número de miembros del Instituto, 
si bien no en cantidad de docentes, si en cantidad de ayudantes de cátedra y/o laboratorio, así como en los médicos agregados e internos ${ }^{31}$. A pesar de haber tenido un breve pasaje, la radicación de Juan Cuatrecasas como investigador exclusivo entre 1937 y $1940^{32}$ incorporó una serie de temáticas de inves- tigación que se acompasaron al viraje del Instituto hacia las terapéuticas biológicas de shock de Sakel y Meduna para el tratamiento de la esquizofrenia -precursoras de las terapias electroconvulsivas- que estaban delimitando el campo de la psiquiatría en el mundo occidental (Perón-Magnan, 2000) ${ }^{33}$.

Tabla 1. Recepción anual de revistas y totales disponibles por país

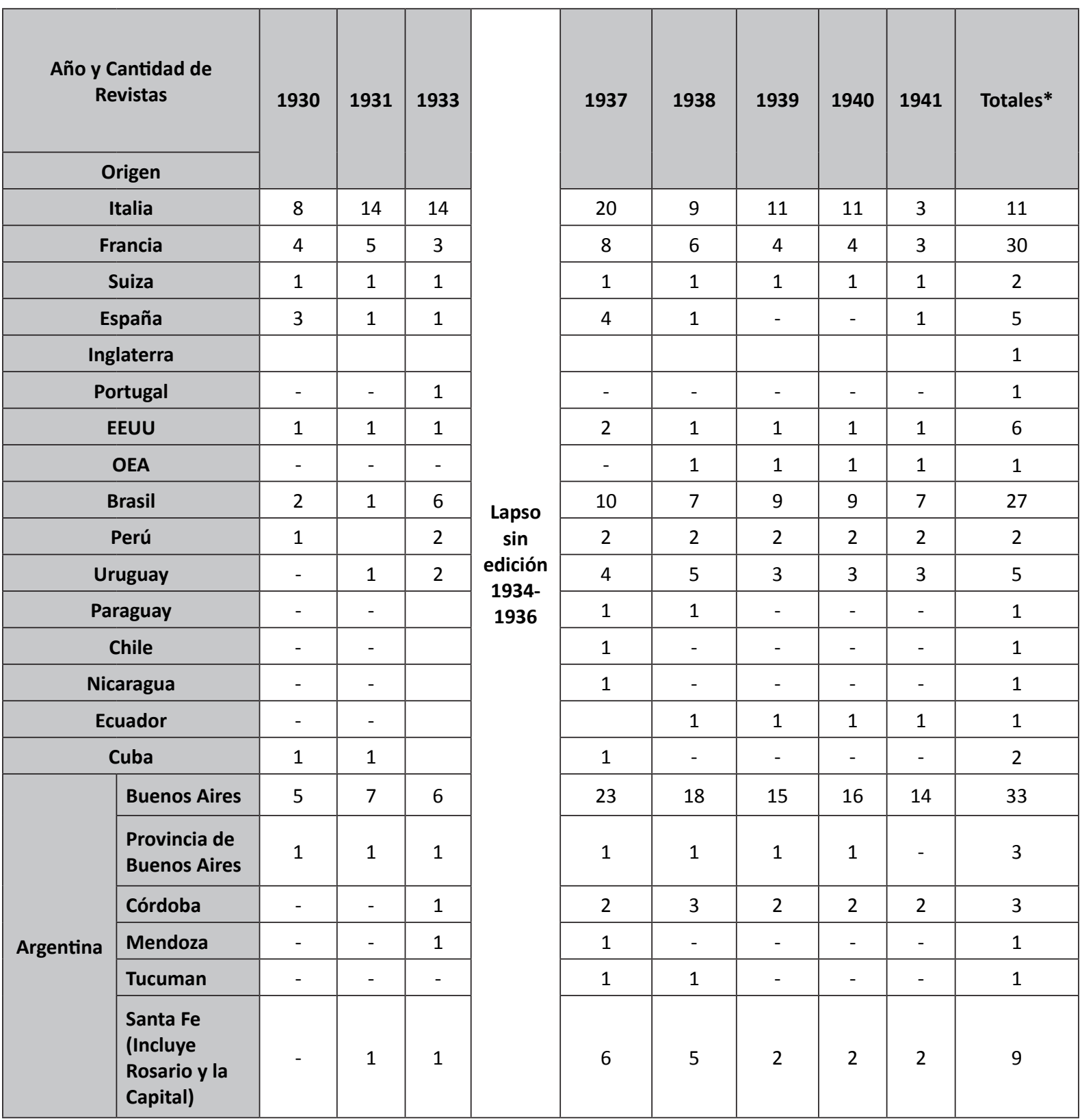

Los totales han sido realizados a partir del cruce entre los datos arrojados por el Boletín del Instituto Psiquiátrico y los disponibles en los registros internos llevados por los psiquiatras, los cuales muestran la recepción de revistas en el período en que no se editó la publicación, así como algunas que no fueron consignadas oficialmente.

Fuente: Boletín del Instituto Psiquiátrico; 1930-1941. 
Tabla 2. Revistas recibidas y períodos de recepción (Europa y Estados Unidos)

\begin{tabular}{|c|c|c|c|}
\hline Nombre de la Revista e Institución de pertenencia & Pais & Ciudad & $\begin{array}{l}\text { Períodos } \\
\text { disponibles }\end{array}$ \\
\hline Annales d' Oculistique & Francia & Paris & 1937 \\
\hline $\begin{array}{l}\text { Annales Medico-Psychologiques Bulletin oficiel de la Societé Médico- } \\
\text { Psychologiques de París }\end{array}$ & Francia & Paris & $1931-1940$ \\
\hline Archives internationelles de Neurologie & Francia & Paris & \\
\hline Clinique et laboratoire & Francia & Paris & \\
\hline $\begin{array}{l}\text { Journal de Medecine de Lyon - Organo des Professeurs, Professeurs } \\
\text { agregés, Medecins des Hospitaux et Medecins practiciens de Lyon }\end{array}$ & Francia & Lion & $1930-1940$ \\
\hline L’Encephale & Francia & Paris & \\
\hline La Presse Medicale & Francia & Paris & \\
\hline La Prophilaxie mentale & Francia & Paris & \\
\hline $\begin{array}{l}\text { La Vie Medicale - Publiant les actes officiels de L'Asociation Generale des } \\
\text { Médecins de France }\end{array}$ & Francia & Paris & $1930-1940$ \\
\hline L'Hygiéne Mentale. Joumal de Psychiatríe apliquée & Francia & & 1933 \\
\hline $\begin{array}{l}\text { Revue Neurologique - Bulletin Officiel de la Societé de Neurologie de } \\
\text { Paris }\end{array}$ & Francia & Paris & $1930-1939$ \\
\hline Annali dell'Ospedale PSichiatrico della provincia de Genova & Italia & Genova & \\
\hline Archivio di Psichologia, Neourologia, Psichiatria e Psicoterapia & Italia & Milano & 1939 \\
\hline $\begin{array}{l}\text { Archivio Fascista di Medicina Politica. Rivista dell' Ospedale Maggiore di } \\
\text { Parma }\end{array}$ & Italia & & $1930-1932$ \\
\hline $\begin{array}{l}\text { Archivio Italiano de Psicología. Instituto de Psicologia Experimental della } \\
\text { Reale Universita }\end{array}$ & Italia & Torino & 1936 \\
\hline $\begin{array}{l}\text { Biochimica e terapia sperimentale. Organo ufficiale della Societá Italiana } \\
\text { di Biochimica }\end{array}$ & Italia & Milano & $1934-1937$ \\
\hline $\begin{array}{l}\text { Bolletino dell'Instituto Sieroterapico Milanese. Publicazione italiana di } \\
\text { Batteriologia ed Inmunologia }\end{array}$ & Italia & Milano & $1932-1940$ \\
\hline Contributi del Laboratorio di Psicologia e Bíología della Universita & Italia & & \\
\hline $\begin{array}{l}\text { Contributti del Laboratorio di Psicologia. Publicazioni della Universitá } \\
\text { Catolica del Sacro Cuore di Milano }\end{array}$ & Italia & Milano & \\
\hline Diffensa Sociale. Rivísta mellsile di igiene, Previdenza e asistenza sociale & Italia & Roma & 1929-1937 \\
\hline $\begin{array}{l}\text { Giornali di Psichiatria e di Neuropatologia. Ospedale Psichiatrico } \\
\text { Provinciale }\end{array}$ & Italia & Ferrara & $1932-1939$ \\
\hline Il Cervello. Giornale di Neurologia & Italia & & \\
\hline L' igiene Mentale. Organo della Liga Italiana di Igiene Mentale & Italia & & 1935 \\
\hline La Giusticia Penale - Rivista de Dottrina, Giurisprudencia e Legislazione & Italia & Roma & $1933-1940$ \\
\hline La liguria medica. Cronica delle Cliniche universitarie di Genova & Italia & Genova & $1931-1935$ \\
\hline L’Ospedale Psíchíatríco. Rivista di Psichiatria, Neurologia e scienza affini & Italia & Napoli & $1933-1937$ \\
\hline $\begin{array}{l}\text { Neopsichiatria. Rasegna di Psichiatria. Neurol. Endocrinologia. Ospedale } \\
\text { Psichiatrico de Volterra }\end{array}$ & Italia & Pisa & $1935-1939$ \\
\hline
\end{tabular}




\begin{tabular}{|c|c|c|c|}
\hline Nombre de la Revista e Institución de pertenencia & Pais & Ciudad & $\begin{array}{l}\text { Períodos } \\
\text { disponibles }\end{array}$ \\
\hline Note e Riviste de Psichiatria. Ospedale Psichiatrico Provinciale di Pesaro & Italia & Pesaro & $1930-1940$ \\
\hline $\begin{array}{l}\text { Rasegna Clinica Scientifica, dell'Istituto Biochimico Italiano sotto il } \\
\text { controllo dello stato }\end{array}$ & Italia & Milano & $1939-1940$ \\
\hline Rasegna de Neurologia Vegetativa & Italia & Firenze & \\
\hline Rasegna di Studi Psichiatrici. Ospedale Psichiatrico di S. Nicole & Italia & Siena & \\
\hline Rassegna di Stiudi Psichiatrici. - Ospedale Psichiatríco S. Nicoló di Siena & Italia & Siena & 1933-1934 \\
\hline Revista de Neurología. Clinica Neurológica & Italia & & \\
\hline $\begin{array}{l}\text { Revista di Psicologia Normale e Patologica. - Organo ufficiale della Societá } \\
\text { Italiana di Psichologia e degli Istituti Universitari di Psichologia Sperimentale }\end{array}$ & Italia & Bologna & 1939 \\
\hline Rivista di Neurologia (Annali di Neurología) & Italia & Napoli & 1930-1937 \\
\hline $\begin{array}{l}\text { Rivista Speriméntale de Freniatria e Medícína Legale delle Alienazioni } \\
\text { Mentali }\end{array}$ & Italia & & \\
\hline $\begin{array}{l}\text { Rivista Speriméntale de Freniatria. Organo della Societá Italiana di } \\
\text { Psichiatria }\end{array}$ & Italia & & $1930-1937$ \\
\hline $\begin{array}{l}\text { Schizofrenie. - Bolletino trimestrale del Primo Centro Provinciale di } \\
\text { Studio della Demenza Precoce }\end{array}$ & Italia & Racconigi & 1935-1939 \\
\hline $\begin{array}{l}\text { Schizofrenie. Ospedale Neuropsichiatrico Provinciale. Primo Centro } \\
\text { Provinciale di Studio della Demenza Precoce }\end{array}$ & Italia & Torino & $1935-1937$ \\
\hline Sperimental de Freniatria. Inst. Psichiatric. Di S. Lazaro & Italia & $\mathrm{s} / \mathrm{l}$ & \\
\hline Terapia. - Publicazione del Instituto Síeroterapico Milanese & Italia & Milano & 1929-1936 \\
\hline Psychological Abstracts - The American Psychological Association & EEUU & Massachutess & $1929-1940$ \\
\hline Baltimore Cornell University Medical Bulletin & EEUU & New York & \\
\hline Endocrinology & EEUU & $\begin{array}{l}\text { Los Angeles, } \\
\text { California }\end{array}$ & \\
\hline The Journal of Educational Psycological House of Warwick & EEUU & & \\
\hline The Psychological Clinic & EEUU & Filadelfia & \\
\hline The Training School Bulletin & EEUU & $\begin{array}{l}\text { Vineland, New } \\
\text { Jersey }\end{array}$ & \\
\hline The International Journal Of Psycho-analysis & Inglaterra & Londres & \\
\hline Travaux de Laboratoire & Portugal & Lisboa & \\
\hline Archivos Españoles de Pediatría & España & Madrid & \\
\hline Clínica y Laboratorio & España & & \\
\hline L’Avenir médical & España & Barcelona & 1936 \\
\hline Le Monde Médical. - Revista Internacional de Medicina y Terapéutica & España & & 1933-1937 \\
\hline Revista de Psicología y Pedagogía & España & Barcelona & 1937 \\
\hline Archives de Psychologie & Suiza & Géneve & 1930-1939 \\
\hline Boletín de la Societé Internationale de Psychologíe et de Psychiatríe & Suiza & Géneve & \\
\hline $\begin{array}{l}\text { Bulletin de la Societe Rumaine de Neurologie, Psychiatrie, Psychologie et } \\
\text { Ednocrinologie (Hospital Socole) }\end{array}$ & Rumania & lasi & \\
\hline Revista de Neuropatología y Psiquiatría & Rusia & Moscú & \\
\hline
\end{tabular}


Tabla 3. Listado de personalidades con las que el Instituto mantenía vínculos

\begin{tabular}{|c|c|c|c|}
\hline Nombre del médico o de la Institución & Institución & Pais & Ciudad \\
\hline Dr. Henri Claude & L'Hygiene Medical & Francia & Paris \\
\hline Institut General Psychologique & & Francia & \\
\hline Dr. R. Cestan & Faculté de Medicine & Francia & Toulousse \\
\hline Dr. J. Abadie & $\begin{array}{l}\text { Clinique des M. Nervouses et mentales. } \\
\text { Faculté de Medecine }\end{array}$ & Francia & Bordeaux \\
\hline Dr. E. Toulousse & Hospital Saint Annte & Francia & Paris \\
\hline Dr. H. Roger & École de Medecine & Francia & Marseille \\
\hline Prof. Doyen Euxiére & Faculté de Medicine & Francia & Montpellier \\
\hline Dr. G. Raviart & Faculté de Medicine & Francia & Lille \\
\hline Dr. M. Segard & & Francia & Paris \\
\hline Prof. Dr. G. Macciotta & $\begin{array}{l}\text { Direttore del Instituto de Clin. Pediátrica de } \\
\text { la Reale Universitá di Caligari }\end{array}$ & Italia & Modena \\
\hline Dr. Agostino Genelli & OFM & Italia & Milano \\
\hline Prof. Dr. M. Selutti & L'Ospedale Psichiatrico & Italia & Napoli \\
\hline Prof. Dr. G. Boschi & $\begin{array}{l}\text { Ospedale Psichiatrico Provinciale - Giornali } \\
\text { di Psichiatria e di Neuropatologia }\end{array}$ & Italia & Ferrara \\
\hline Prof. Dr. Ch. Ladame & Faculte de Medicine & Suiza & Genéve \\
\hline Dr. E. Claparéde & & Suiza & Genéve \\
\hline Prof. Dr. E. Bleuler & Clinique Psychiatrique & Suiza & Zurich \\
\hline Prof. Dr. Pierre Bovet & & Suiza & Zurich \\
\hline Dr. J. Larguier des Bancels & & Suiza & Lausanne \\
\hline Dr. Gregorio Marañón & Facultad de Medicina & España & Madrid \\
\hline Prof. L. E. Mira & & España & Barcelona \\
\hline Dr. José Fariña & & España & $\begin{array}{l}\text { Santa Cruz de } \\
\text { Tenerife }\end{array}$ \\
\hline Prof. W. M. Dougall & Harvard University & EEUU & $\begin{array}{l}\text { Cambridge } \\
\text { Masachussets }\end{array}$ \\
\hline Dr. José Trueta & & Inglaterra & Londres \\
\hline Prof. Dr. Graig & Faculty of Medicine & Inglaterra & Londres \\
\hline Prof. Dr. Robertson & Faculty of Medicine & Escocia & Edinburgh \\
\hline Prof. D. De Moor & & Bélgica & Gand \\
\hline Dr. A. Ley & & Bélgica & Bruxelles \\
\hline Prof. Dr. F. D’Hollander & Faculté de Médecine & Bélgica & Lovaine \\
\hline Dr. L. A. Kwint & Institut Psychoneurologique & Rusia & (Ukranie, Kharcov) \\
\hline Prof. Dr. A. Heymanovitch & Institut Psychoneurologique & Rusia & (Ukranie, Kharcov) \\
\hline Prof. Dr. Marinescu & Facultad de Medicina & Rumania & Bucarest \\
\hline Dr. L. von Meduna & & Hungria & Budapest \\
\hline
\end{tabular}


El dieciocho de febrero de 1938, el Instituto Nacional de Clasificación y Criminología de la Penitenciaría de Santiago -dependiente de la Dirección General de Prisiones de Chile-, dirigía una nota a la redacción del Boletín para notificar el envío de los Archivos Chilenos de Criminología. Allí manifestaba la voluntad de insertarse en redes científicas latino-americanas, solicitaba el envío de los números anteriores disponibles del BIP y consultaba por la posibilidad de ser reseñados en éste. A principios de 1940, el Dr. Veiga de Caravahlo inaugura Neuronio. Arquivos Latino-Americanos de Neurologia, Psiquiatria, Medicina Legal y ciencias afins, y por este motivo escribe desde San Pablo al comité editor del BIP, para comentar dicha novedad editorial, como también su interés por establecer vínculos con otros Institutos. El 15 de marzo del mismo año, el Dr. Antonio Menéndez Crespo dirige una carta al secretario de redacción del BIP desde La Sierra, Cuba, requiriendo el precio de suscripción de la revista, dado su interés por acceder regularmente a la misma al haber tomado contacto con ésta gracias a un colega. En respuesta a su pedido, Esteban Carro le informa el 20 de agosto que la publicación del Boletín tiene por único fin el intercambiado con otras revistas, ante lo cual le envía igualmente el último número ${ }^{34}$.

La "segunda época" del Instituto incluyó un nuevo período para el Boletín, que se insertaba en un nuevo contexto internacional, donde el conflicto bélico mundial redujo sustantivamente el flujo de publicaciones europeas. Esto dio cuenta, asimismo, de un proceso de partida doble que mostraba el redireccionamiento de la circulación junto a una nueva posición del Instituto, y con ello se desplazó el centro de gravedad del canje. Por una parte, de la primacía italiana, el Instituto amplió sus márgenes hacia espacios "ex-céntricos", tales como Bélgica, Hungría, Rumania o Rusia, pero de manera inversa a lo acontecido en el pasado, puesto que el contacto surgió desde dichos países. Por caso, a comienzos de 1939, la Sociedad de Salud Pública de Hungría escribió al Instituto para enviar el número 5 de su revista ("La Higiene") y solicitar el envío de los boletines en intercambio, con la intención de profundizar las relaciones entre "la ciencia española y la húngara" (Ver imagen $n^{\circ} 5$ ). El cinco de julio del mismo año, el comité de redacción de la Revue Belge de Sciences Medicales se dirigió al comité del BIP para comenzar a vincularse con la publicación (Ver imagen $\left.n^{\circ} 3\right)$, y en 1941, el ocho de enero la Societe pour les Relations Culturelles entre I'URSS et le pays etrangers contactó al Instituto para vincularlo regularmente a la biblioteca del Hospital Psiquiátrico de Moscu (Ver imagen $n^{\circ} 4$ ). El 7 de noviembre del mismo año, el
Sindicato Español Universitario solicitaba el envío de los boletines por estar reorganizando la Biblioteca de Internos del Hospital Provincial de Madrid. Otros contactos con el viejo continente, mientras, estuvieron ligados a iniciativas locales por recepcionar y poner en práctica nuevas terapéuticas. Tal fue el caso con las terapias convulsivantes a partir de comas insulínicos y cardiazólicos para el tratamiento de la esquizofrenia, perseguidos con el objeto de agilizar el paso de los enfermos por el hospicio, e implementados a partir de publicaciones de Manfred Sakel y Lazlo Von Meduna en 1935 y 1936 respectivamente ${ }^{35}$.

Ahora bien, en este desplazamiento en el eje del canje se incrementó de manera sostenida el intercambio de publicaciones en el ámbito regional, en especial con Brasil y Buenos Aires, lo cual evidenciaba un mayor reconocimiento de la actividad del Instituto en el campo local. Mientras que en los inicios del Boletín se observaba un reconocimiento en función de la apelación de vínculos que Ciampi o el grupo de psiquiatras porteños podía activar, en su segunda época la difusión de su producción científica se encontraba autonomizada, ora por la circulación en congresos y reuniones, ora por la pertenencia a sociedades científicas, la reseña de sus artículos en revistas especializadas adquiría otro nivel (Ver tabla 4).

En cuanto a su actividad académica, en el mes de septiembre de 1934 se llevó a cabo en Rosario el V Congreso Nacional de Medicina, donde los grupos psi participaron activamente. El equipo del Instituto participó con cinco presentaciones, ${ }^{36}$ mientras que Fracassi y sus colaboradores hicieron lo propio con siete trabajos. A esto debemos agregar la presencia de algunos personajes con los cuales los miembros del Instituto de Psiquiatría compartían redes, como Nerio Rojas, José C. Belbey, Arturo Rossi y Leopoldo Bard.

A partir de 1937, los psiquiatras fueron convocados por Raimundo Bosch para su Escuela de Médicos Legistas -una instancia para la formación de peritos médicos gestada dos años antes (Bosch, 1966)-, donde Antonio Foz dictaba cursos de Clínica Psiquiátrica y Psicopatología forense, mientras que Arturo Bruno hacía lo propio con Toxicología y Química Legal. Esto no sólo constituyó una oportunidad de desempeño y reconocimiento, sino también la posibilidad para los psiquiatras para dirigir trabajos de investigación e informes médico legales de los alumnos ${ }^{37}$. La participación de los miembros del Instituto, sin embargo, no se resumió allí solamente, ya que muchos decidieron formarse como peritos en los años sucesivos ${ }^{38}$. 


\section{Imagen 3}

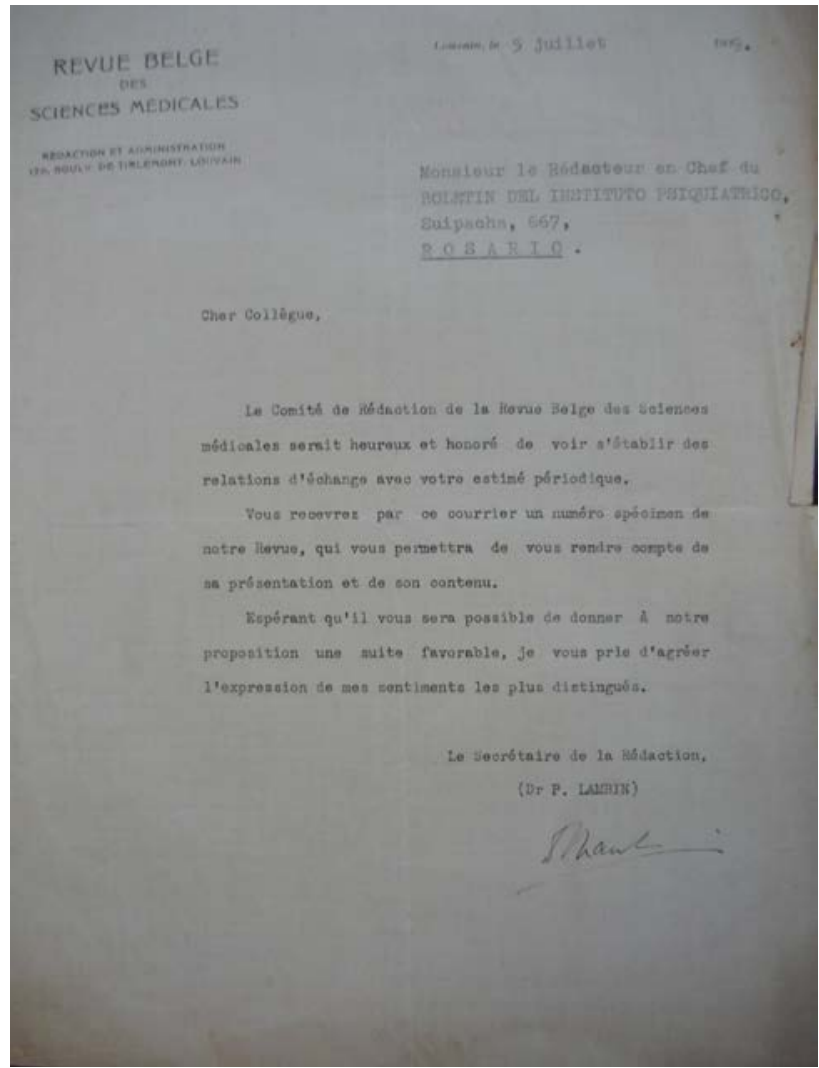

Imagen 4

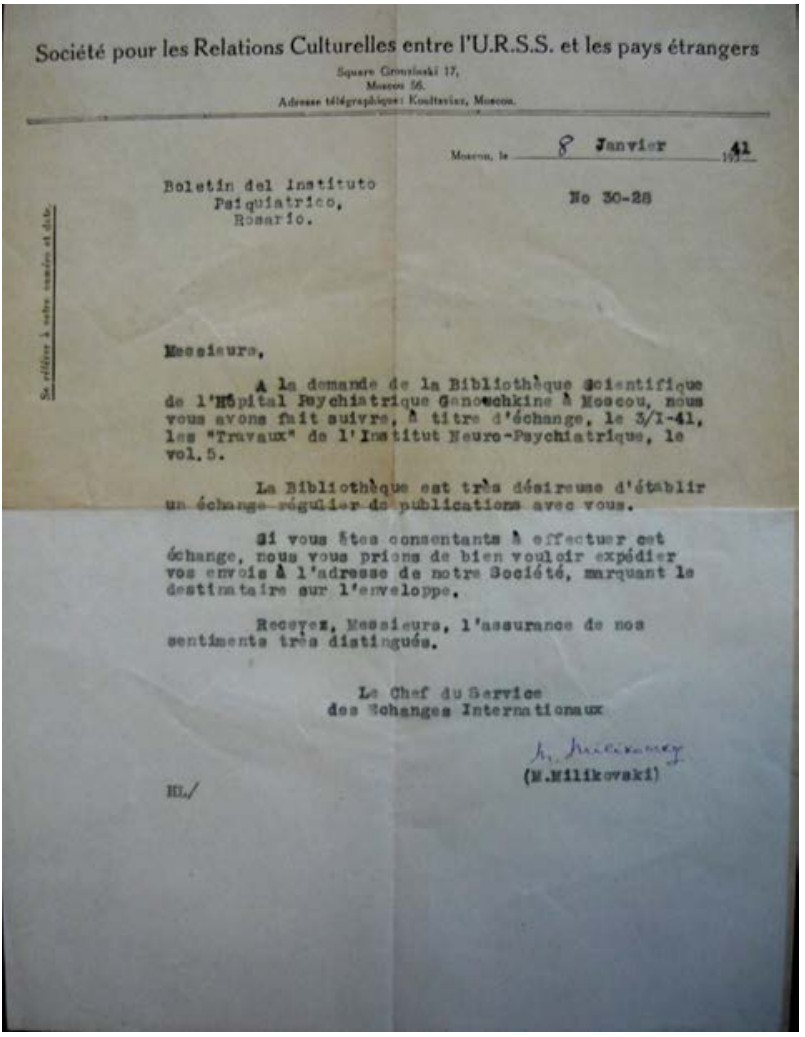




\section{Imagen 5}

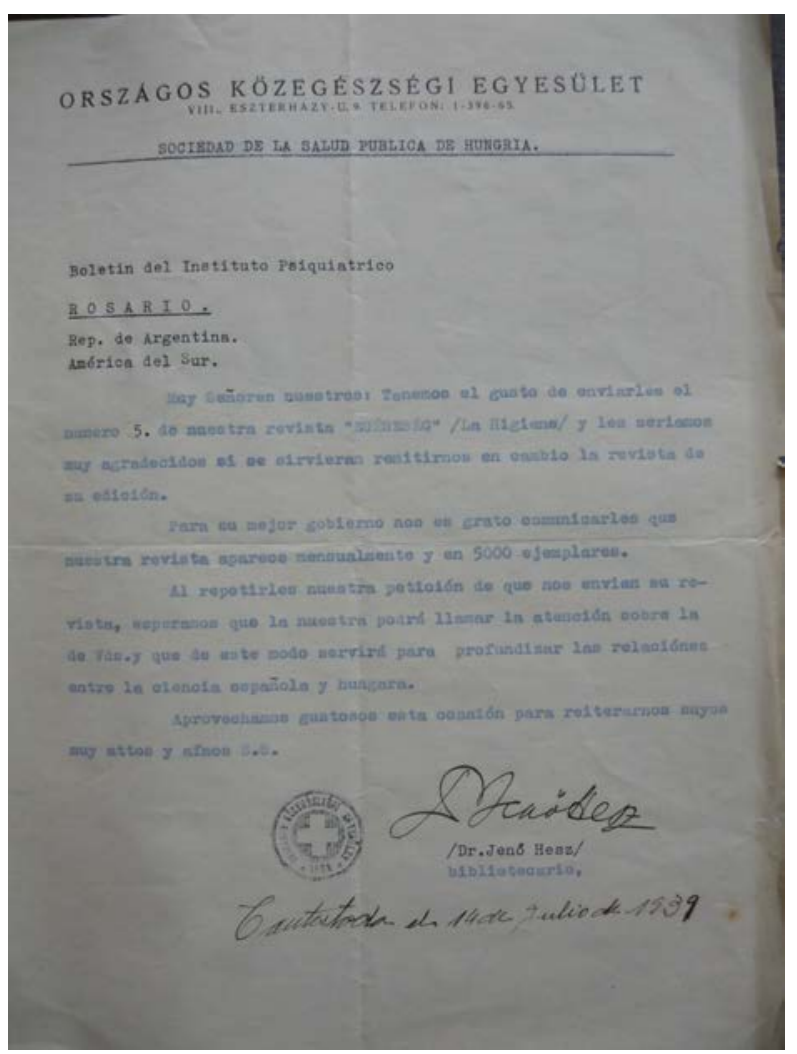

Tabla 4. Revistas recibidas y períodos de recepción (América Latina)

\begin{tabular}{|c|c|c|c|}
\hline Nombre de la Revista e Institución de pertenencia & Pais & Ciudad/Partido & $\begin{array}{l}\text { Períodos } \\
\text { disponibles }\end{array}$ \\
\hline Anales da Facultade de Medicina da Universidade de Sao Paulo & Brasil & Sao Paulo & 1934 \\
\hline Archivos Brasileros de Andrología & Brasil & Rio de Janeiro & 1939 \\
\hline Archivos Brasileros de Higiene Mental & Brasil & Rio de Janeiro & \\
\hline Archivos Brasileros de Neuriatria e Psiquiatria & Brasil & Rio de Janeiro & $1931-1937$ \\
\hline Arquivo do manicomio judiciario Hector Carrilho & Brasil & & \\
\hline Arquivos Brasileros de Hygiene Mental & Brasil & & \\
\hline Arquivos da Assietncia a Psicopatas do Pernambuco & Brasil & Pernambuco & $1932-1935$ \\
\hline Arquivos da Policia Civil de Sao Paulo & Brasil & & \\
\hline Arquivos da Sociedade de Medicina Legal e Criminologia de Sao Paulo & Brasil & Sao Paulo & $1931-1936$ \\
\hline Arquivos de Neuro Psiquiatria & Brasil & & \\
\hline Arquivos do Servicio de Assitencia a Psicopatas do Estado de Sao Paulo & Brasil & Sao Paulo & $1938-1940$ \\
\hline $\begin{array}{l}\text { Bahia Medica. Órgano oficial do Instituto Brasileiro para Investicao da } \\
\text { tuberculose }\end{array}$ & Brasil & & 1932-1939 \\
\hline Boletim da Hygiene Mental & Brasil & & \\
\hline Boletim da Sociedade de Medicina e Cirugia de Sao Paulo & Brasil & Sao Paulo & $1930-1940$ \\
\hline Boletim de Secretaria Geral de Saùde e Assietncia & Brasil & & \\
\hline
\end{tabular}




\begin{tabular}{|c|c|c|c|}
\hline Nombre de la Revista e Institución de pertenencia & Pais & Ciudad/Partido & $\begin{array}{l}\text { Períodos } \\
\text { disponibles }\end{array}$ \\
\hline Boletin da Secretaria General de Saude e Asistencia & Brasil & Rio de Janeiro & \\
\hline Imprensa Médica. Revista del Instituto Brasileiro de Hormoterapia & Brasil & & 1936 \\
\hline La Odontología. Revista Ibero Americana de Ciencia Dental & & & $1934-1935$ \\
\hline Memorias do Hospital du Juquery & Brasil & & \\
\hline $\begin{array}{l}\text { Neurobiologia. Organo oficial da Sociedade de Psiquiatria, Neurologia e } \\
\text { Higiene Mental do Nordeste Brasilero }\end{array}$ & Brasil & Recife, Pernambuco & $1938-1940$ \\
\hline $\begin{array}{l}\text { Neurônio - Arquivos latino-americanos de neurologia, psiquiatria, } \\
\text { medicina legal e ciências afins }\end{array}$ & Brasil & Sao Paulo & \\
\hline Revista brasileira de Psiquiatria & Brasil & & \\
\hline Revista de Criminologia e Psiquiatria de Sao Paulo & Brasil & Sao Paulo & \\
\hline Revista de Neurologia e Psiquiatria de Sao Paulo & Brasil & Sao Paulo & $1934-1940$ \\
\hline Revista do Associacao paulista de Medicina & Brasil & Sao Paulo & $1932-1936$ \\
\hline Revista Medica de Minas. Organo del Sindicato Médico de Belo Horizonte & Brasil & Belo Horizonte & 1940 \\
\hline $\begin{array}{l}\text { Revista medico municipal - Publicacao de Secretaria Geral de Saùde e } \\
\text { Assistencia de Prefetura do Distrito Federal }\end{array}$ & Brasil & & \\
\hline Sao Pablo Medico & Brasil & Sao Paulo & $1938-1940$ \\
\hline $\begin{array}{l}\text { Archivos Uruguayos de Medicina, Cirugía y especialidades. Órgano oficial } \\
\text { de las Sociedades Médico-Científicas del Uruguay }\end{array}$ & Uruguay & Montevideo & $1933-1940$ \\
\hline Asociación Sindical. Sindicato Médico del Uruguay & Uruguay & Montevideo & $1934-1940$ \\
\hline Boletín del Instituto Internacional Americano de Protección a la Infancia & Uruguay & Montevideo & $1930-1940$ \\
\hline Revista de Neurología, Psiquiatría y Medicina Legal & Uruguay & Montevideo & \\
\hline $\begin{array}{l}\text { Revista de Psiquiatría del Uruguay. Órgano oficial de la Sociedad de } \\
\text { Psiquiatría }\end{array}$ & Uruguay & & $1935-1940$ \\
\hline La Crónica médica & Perú & Lima & $1929-1940$ \\
\hline Revista Médica Peruana. Vocero Oficial de la Asociación Médica Peruana & Perú & Lima & $1930-1940$ \\
\hline Revista de Neurología y Psiquiatría de la Habana & Cuba & La Habana & $1929-1930$ \\
\hline Revista de Psiquiatría y Neurología. Hospital de Mazorra & Cuba & & \\
\hline Revista Médica. Organo de la Sociedad Médica de Managua & Nicaragua & & $1933-1934$ \\
\hline $\begin{array}{l}\text { Boletín de la Dirección General de Protección de Menores. Revista de } \\
\text { Psicología, Pedagogía, Derecho y Psiquiatría }\end{array}$ & Chile & Santiago de Chile & $1929-1936$ \\
\hline $\begin{array}{l}\text { Archivos de Criminología, Neuropsiquiatría y Disciplinas conexas. Órgano } \\
\text { del Instituto de Criminología de Quito }\end{array}$ & Ecuador & Quito & $1937-1939$ \\
\hline Boletín de la Sociedad de Medicina y Cirugía del Paraguay & Paraguay & & 1936 \\
\hline
\end{tabular}

Durante 1937, Lanfranco Ciampi y Antonio Foz dictaron una serie de conferencias. El primero lo hizo en el Ateneo de la Facultad de Medicina del Litoral, en el Consorcio de Médicos Católicos y en la Asociación de intelectuales, Artistas, Periodistas y Escritores-AIAPE- ${ }^{39}$; mientras que Foz, por su parte, hizo lo propio en el Consorcio de Médicos Católicos -conti- nuadas a lo largo de los años-, así como una divulgación radiofónica "a pedido" de la Dirección General de Higiene ${ }^{40}$. Nos interesa destacar la participación de miembros del Instituto en la AIAPE, no sólo porque dos destacados miembros de esta última, como Gregorio Bermann y Nerio Rojas, tenían amplios vínculos con el Instituto, sino porque también marca una 
pauta sobre el grado de apropiación de las propuestas teóricas más radicales del fascismo europeo como de aquellas formuladas en el plano local ${ }^{41}$. El Instituto de Psiquiatría canjeaba su boletín con organismos italianos abiertamente fascistas y, sin embargo, estos vínculos no obturaban su capacidad de leer críticamente las propuestas extremistas desarrolladas en Europa, tal como Foz lo expresó en su artículo "Eugenesia” de 1938, donde revisitó las principales posturas al respecto, para concluir en claro repudio a las tendencias "negativas"42.

En términos de sociabilidad académico-cultural -y junto con el estrechamiento de los lazos entre ambos grupos con la Escuela de Médicos Legistas-, se constituyó en 1937 la Sociedad de Psiquiatría, Psicopatología y Medicina Legal, donde confluían el Instituto Psiquiátrico con el de Medicina Legal. Presidida por Lanfranco Ciampi y Raimundo Bosch, sus integrantes eran los mismos miembros del Instituto dirigido por el psiquiatra italiano, a quienes debe sumarse los socios titulares: Gonzalo Bosch, Sebastian Soler ${ }^{43}$ y Juan Cuatrecasas ${ }^{44}$. La trayectoria de esta nueva asociación tiene una presencia mucho más rastreable en el Boletín, dado que aquí se publicaban los resultados de las investigaciones presentadas en cada reunión. Esto representa también un cambio en la concepción de la investigación científica, dado que anteriormente el Boletín sólo publicaba los trabajos de manera directa, incorporando así un ámbito previo de discusión y supervisión de resultados. En el mismo año, Teodoro Fracassi fundó la Sociedad de Neurología, Psiquiatría y Especialidades Afines de Rosario con su grupo, cuyo antecedente era la Revista Argentina de Neurología y Psiquiatría.

En 1938 se celebraron dos eventos académicos donde participaron los miembros del Instituto. Las Jornadas Sudamericanas de Medicina y Cirugía en la ciudad de Montevideo, a las cuales asistieron Antonio Foz y Arturo Bruno, y el VI Congreso Nacional de Medicina en Córdoba, donde se presentaron siente trabajos que, por otro lado, marcaban el claro cambio de rumbo en las investigaciones ${ }^{45}$. Este mismo congreso ofreció también a los psiquiatras una oportunidad para jugar una carta en su legitimación regional, al exponer su reciente vinculación con Ladislao Von Meduna, con quien Foz elaboró un trabajo en coautoría a pedido del médico húngaro (Meduna y Foz, 1938, p. 168).

En 1938 y 1940, por otra parte, el Instituto recibió visitas de George Dumas, Ladislao von Meduna, y Emilio Mira y López ${ }^{46}$, quien además sostenía un vínculo con Cuatrecasas por su formación doctoral compartida, así como con Juan Lazarte, quien convocó a ambos (Ku- rowski, 2001). Pero Mira asimismo resultaba una referencia central en el plano local no sólo teóricamente, sino por haberse instalado temporalmente en la provincia de Santa Fe, años más tarde, cuando se le entregó la Dirección estatal de los servicios de Salud Mental.

Si bien no lo abordaremos aquí, un aspecto central en el crecimiento de la legitimidad del Instituto fue su rol en la incorporación de la higiene mental como saber de estado en la nueva estructura ministerial de la provincia de Santa Fe (Allevi, 2016). En efecto, desde los inicios del Instituto, los psiquiatras pugnaron en diversas instancias estatales por obtener mayores recursos, y sobre todo por la construcción de una colonia de alienados, pieza central para completar el dispositivo de atención en el higienismo mental. Esta apelación a los poderes públicos se realizó inicialmente desde la vía de la sociabilidad, apelando a los vínculos en alguna agencia municipal, provincial o incluso nacional. Sin embargo, con las transformaciones en la estructura política provincial durante de la segunda mitad de la década del treinta, la concepción de nuevas obras públicas en salud desde el Departamento de Salud Pública (1939) -luego Ministerio (1941)- incluyó la perspectiva de la higiene mental sin que mediase una intervención explícita de los psiquiatras rosarinos. Sostenemos que ello se debió a la constitución de una lógica autonomizada en la formación de especialistas, dado que el ministro de salud provenía de la Facultad de Ciencias Médicas de Rosario.

\section{REFLEXIONES FINALES: EN TORNO A LA CONSTRUCCIÓN DE UN ESPACIO PROPIO}

En esta dinámica de conjunto, hemos rastreado una faceta particular de un proceso trasnacional: la profesionalización disciplinar de la psiquiatría respecto a la medicina y a la neurología en particular en el caso de la ciudad de Rosario, uno de los pocos centros de atención psi de Argentina durante la entreguerras. Entre sus estrategias para fortalecer su estatuto de cientificidad y autonomía, revisamos el intercambio intelectual, los espacios de sociabilidad científica que fueron cristalizando a lo largo de los '30, y las redes generadas a partir de la recepción de revistas nacionales e internacionales, con lo cual, además, se leían y apropiaban nuevas ideas, al tiempo que difundían las producciones locales.

En líneas generales podemos ver que en la "primera época" del Instituto, la participación en eventos responde a una clave bifronte: tanto académica como política: si bien hay un claro intento de dar a conocer sus producciones en un contexto más amplio -y así lo 
demuestra el caudal de presentaciones en los distintos eventos a los que asistieron-, también es cierto que en los primeros años de su existencia, y más aún en los eventos donde coincidían, se tradujo una cierta rivalidad con el grupo de neurólogos rosarinos, desplazados de la dirección del Instituto; desde allí puede pensarse el patrón de presentaciones múltiples por parte de Teodoro Fracassi. Por otro lado, los intercambios de revistas a partir del canje respondieron en este primer momento a un eje de relación fundamentalmente con Europa, y en especial con Italia, a partir de la figura de Ciampi como mediador.

Ahora bien, ya en su "segunda época", el Instituto demostraba una mayor autonomía en lo referido a su posicionamiento en un campo psiquiátrico cada vez más definido en términos locales e internacionales. A la reorientación del eje de circulación con países europeos en el contexto bélico se agrega la expansión del intercambio con Brasil, junto a las solicitudes que comienzan a llegar al comité de redacción para recibir la publicación. Ello da cuenta del lugar propio que disponían en el mundo académico, así como de los resultados de su participación en distintas instancias sociabiliares desde principios de la década. Debemos agregar a esto el crecimiento del Instituto en cantidad de miembros y sus producciones luego del cambio

\section{NOTAS}

1. Discípulo de Sante de Sanctis entre 1913 y 1919, este médico se instala en Argentina en 1920, donde logró vincularse estrechamente con algunos personajes relevantes del campo psi en formación, siendo contratado como asesor por el Consejo Nacional de Educación, y luego por Antonio Agudo Ávila como docente en la nueva Facultad de Rosario. Correspondencia Ciampi-De Sanctis- Archivio di Storia della Psicologia. Fondo Sancte de Santis, Universidad de Roma, 23/3/1920; 2/5/1920, 11/5/1920.

2. Cabe destacar que en Argentina, la amplia mayoría de las Universidad son de gestión estatal nacional, apareciendo casas de altos estudios privadas luego de la segunda mitad del siglo XX. De allí la posibilidad de las autoridades de gobierno de intervenir estos espacios a pesar de la autonomía proclamada con la Reforma de 1918.

3. Arturo Ameghino (1880-1949) fue un médico argentino especializado en Neurología y Psiquiatría entre 1911 y 1914 en París y Montpellier (Francia). En 1931 accede a la titularidad de la cátedra de Clínica Psiquiátrica en la Universidad de Buenos Aires, y desde ese momento se observa su progresiva constitución en una figura de peso del campo científico. Fue profesor de Patología Social en la Escuela de Servicio Social de la UBA, presidente de la Sociedad de Neurología y Psiquiatría y miembro fundador en su dirección. En este sentido, la incorporación de Cuatrecasas permitió orientar las investigaciones hacia terapéuticas de corte endocrinológico, sumando a esto los tratamientos convulsivantes adoptados sobre fines de la década, con lo cual especializaban su disciplina en los lineamientos internacionales.

En suma, este acotado recorrido buscó evidenciar una miríada de actividades y esferas de acción académicos donde los actores pugnaron por la legitimidad de su ejercicio profesional, las cuales deben combinarse con el plano de la política, la acción en la esfera pública y sus vínculos con el Estado como instancia clave de legitimación, cuestión abordada en otros trabajos.

\section{AGRADECIMIENTOS}

Deseo expresar mi agradecimiento a los evaluadores de este trabajo, que me permitieron revisarlo y pulirlo a la luz de sus críticas enteramente constructivas. Asimismo, a la directora de la carrera de especialización en Psiquiatría y docente de la Facultad de Ciencias Médicas de la Universidad Nacional de Rosario (Argentina), Dra. Analía Ravenna, por permitirme acceder al archivo disponible en la Biblioteca de Psiquiatría de dicha unidad académica, y consultar de forma irrestricta los documentos allí contenidos, aún no catalogados. de la Sociedad de Medicina Legal y Toxicología -a la cual Ciampi también pertenecía-.

4. Esta Conferencia se originó en reuniones sostenidas en la Sociedad de Neurología y Psiquiatría de Buenos Aires desde 1926, propuesta originalmente por el psiquiatra chileno Hugo Lea Plaza. Su concreción desató una serie de tensiones al interior de dicha Sociedad por la pugna entre disciplinas (Neurología y Psiquiatría), amén de las cuales, cabe señalar la movilización de recursos que implicaron: auspicios en dos ministerios nacionales, la obtención de recursos de la Asociación Médica Argentina, el Municipio de la Capital, el Gobierno de la Nación, y el Jockey Club de Buenos Aires, además de las actividades "recreativas" organizadas en paralelo, una de las cuales estuvo a cargo de la Gobernación de la provincia de Buenos Aires. La trascripción de las jornadas fue realizada por los taquígrafos de la Cámara de Diputados de la Nación. Ameghino, Arturo (1929), Actas de la Primera Conferencia Latino Americana de Neurología, Psiquiatría y Medicina Legal. Buenos Aires: Imprenta de la Universidad. Tomo I, p.29.

5. En las palabras de inauguración de la Conferencia, Ameghino plantea esta disputa: "Es de ese modo que la propia ciencia médica se asocia al prejuicio social para conspirar sin quererlo contra la Psiquiatría; y es por ello que el psi- 
quiatra, peregrino en las clínicas según el público astuto, y en realidad paria en el seno de la profesión, cede a su impotencia, déjase invadir por la quietud, y conspira él también con su silencio contra los intereses generales." Ameghino, Arturo (1929), Actas de la Primera Conferencia Latino Americana de Neurología, Psiquiatría y Medicina Legal. Buenos Aires: Imprenta de la Universidad. Tomo I, pp. 40-41).

6. La presencia de éste último médico - presidente de la Liga Brasilera de Higiene Mental- no debe pasarse por alto, en tanto Gonzalo Bosch fundó dicha institución en Buenos Aires al año siguiente, sumando a ello que la siguiente Conferencia se realizó en Brasil. Véase infra.

7. Cayetano Viale fue un médico italiano convocado "por intermedio de la cancillería y el ministro plenipotenciario de la legación argentina en Roma" para ocupar la dirección del Instituto de Fisiología y su cátedra entre 1926 y 1929 (Bosch, 1966, p. 124).

8. Gregorio Bermann (1894-1972) fue un médico psiquiatra y licenciado en filosofía y letras de la provincia de Córdoba. Activo partícipe de la Reforma Universitaria durante y miembro del Partido Comunista en sus últimos años, se desempeñó como docente universitario, hasta ser cesanteado en 1936 de su cátedra, cuando ingresa a la AIAPE, con cuyo director, Aníbal Ponce, guardaba amistad. Participó, además, como médico en el frente republicano de la Guerra Civil Española en 1937. (Tarcus: 2007, pp. 61-63).

9. “Informaciones". Boletín del Instituto Psiquiátrico (1929), Año I (2), pp. 128-129.

10. El Centro de estudiantes repudió abiertamente el concurso, destacando los antecedentes de Bermann no sólo en la materia, sino en el desempeño de la docencia universitaria, explicitando que el obstáculo señalado por el jurado evaluador se resumía en su militancia comunista. Revista El Ateneo (1933), p. 37.

11. "Liga Argentina de Higiene Mental". Boletín del Instituto Psiquiátrico (1929), Año I (3), pp. 221-226.

12. "Segunda Conferencia Latino-americana de Neurología, Psiquiatría y Medicina Legal". Boletín del Instituto Psiquiátrico (1930), Año II (5), p. 172.

13. "Segunda Conferencia Latino-americana de Neurología, Psiquiatría y Medicina Legal". Boletín del Instituto Psiquiátrico (1930), Año II (5), p. 173.

14. "Sociedad de Neurología y Psiquiatría de Buenos Aires". Boletín del Instituto Psiquiátrico (1931), Año III (10-11), pp. 204-206.

15. "Noticias y Comunicaciones". Revista de Criminología, Psiquiatría y Medicina Legal (1930), Año XVII (99), p. 374.

16. La Sociedad de Psicología de Buenos Aires surge como iniciativa de Enrique Mouchet, quien en ese momento se desempeñaba como profesor de Psicología Experimental y Fisiología en la Facultad de Filosofía y Letras de la Universidad de Buenos Aires, y que será su presidente. Entre los participantes encontramos como vice a Osvaldo Loudet, José C. Belbey como secretario, José L. Alberti como tesorero y sus vocales: Víctor Mercante, Coriolano Alberini y Arturo Ameghino. "Notas y comentarios". Revista de Criminología, Psiquiatría y Medicina Legal (1930), Año XVII, (100), p. 495.

17. Revista de Criminología, Psiquiatría y Medicina Legal (1930), Año XVII, (102), p. 765.

18. Correspondencia Ciampi-De Sanctis- Archivio di Storia della Psicologia. Fondo Sancte de Santis, Universidad de Roma, 3/12/1923, 7/2/1928.

19. "Informaciones". Boletín del Instituto Psiquiátrico (1929), Año I (2), p. 126

20. Este especialista era una figura de peso en el campo de la Psicología francesa, no sólo por la relevancia de sus obras, sino por haber sido el sucesor de Ribot en el Collége de France.

21. "Las jornadas rioplatenses de Neuropsiquiatría". Boletín del Instituto Psiquiátrico (1932), Año IV (12-15), p. 90.

22. "Una distinción a nuestro director". Boletín del Instituto Psiquiátrico (1932), Año IV (12-15), p. 85.

23. "Sociedad Argentina de Criminología”. Revista de Criminología, Psiquiatría y Medicina Legal (1934), Año XXI (122), pp.58-68.

24. "Análisis de libros y revistas". Revista de Criminología, Psiquiatría y Medicina Legal (1930) Año XVII (97), pp. 112113; Año XVII (98), p. 243.

25. A saber, Gonzalo Bosch, Antonio Foz, Benito V. Casanova (Sub director del hospital, fallecido en 1929), Carlos crespo, José M. Cid, José A. Alberti y Arturo Bruno. E 1932, Troilo pasa a formar parte del comité de redacción, junto con Inocencio Ansaldi (adscripto y medico agregado a la cátedra de Psiquiatría, respectivamente), mientras que Juan B. Vázquez (nuevo jefe de clínica Psiquiátrica) ocupa la secretaría, que en 1934 volverá a ser ocupada por Troilo.

26. "A guisa de prólogo". Boletín del Instituto Psiquiátrico (1937), Año I, 2da época (20-21), p. 5. No lo analizaremos aquí, pero Teodoro Fracassi tuvo su cuota de participación en dicha intervención, la cual no arrojó ninguna irregularidad.

27. Biblioteca de la Cátedra Psiquiatría de Adultos. Facultad de Ciencias Médicas. Universidad Nacional de Rosario, 7/10/1935 y 15/10/1935, respectivamente.

28. Biblioteca de la Cátedra Psiquiatría de Adultos. Facultad de Ciencias Médicas. Universidad Nacional de Rosario.

29. "Facultad de Ciencias Médicas, Farmacia y Ramos Menores. Consejo Directivo. Sesiones". Boletín de la Universidad del Litoral (1935), Año IX, pp. 113-114.

30. "Facultad de Ciencias Médicas, Farmacia y Ramos Menores. Consejo Directivo. Sesiones". Boletín de la Universidad del Litoral (1936), Año X, p. 147. 
31. Tomas Ocaña (Prof. Adjunto de Anatomía Patológica y Jefe del Laboratorio de Histopatología), Nicolás Vita (Medico Interno), Pedro Nisibar (Medico agregado). Junto a ello, la reincorporación de Carlos Crespo (como Medico agregado), mientras que Troilo - ahora profesor Adjunto de Clínica Psiquiátrica y vicedirector del Hospital- continuaba en la Secretaría de redacción. Durante la segunda mitad de 1938 se incorporan cuatro miembros: Aquilino Rodríguez Gallo (Médico agregado), Zoe Nélida Bula (Médica Agregada), Jorge E. Camoirano (Ayudante del Laboratorio de Bio química), y Maria E. Ansiaume (Ayudante principal del mismo Laboratorio). A partir de 1941, Maria del Carmen Foz comienza a desempeñarse como ayudante en el Laboratorio de Psicología Experimental, que continuaba sin designación de jefe.

32. Juan Cuatrecasas fue un médico, recibido en la Universidad de Barcelona, que formó parte del innumerable contingente de científico que llegó a la Argentina al desatarse la Guerra Civil española. A su venida en 1937, fue nombrado encargado de investigaciones del Instituto -el equivalente a un investigador categoría full contemporáneoBoletín del Instituto Psiquiátrico, 1937). Antonio Gentile señala agudamente que la temática de investigación de Cuatrecasas -glándulas de secreción interna y sus hormonas- era fundamental en la delimitación de un campo propio de la Psiquiatría para distanciarse de la Neurología, un aporte que el Instituto de Psiquiatría supo aprovechar, a pesar del breve paso de Cuatrecasas por el mismo (Gentile, 2003, pp. 75-77).

33. El abordaje de la recepción y práctica de las mismas en otro hospital de Argentina, véase Golcman (2017).

34. Biblioteca de la Cátedra Psiquiatría de Adultos. Facultad de Ciencias Médicas. Universidad Nacional de Rosario.

35. La incorporación de esta terapéutica de la esquizofrenia incluyó un desarrollo de estrategias internas para poner en práctica los nuevos métodos con nuevos agentes convulsivantes, dado el precio del cardiazol y la insulina. Pero además, en 1938 el director del Hospital contactó a Ladislaz Von Meduna, con quien publicaron en conjunto un trabajo sobre su terapéutica de la esquizofrenia.

36. Actas del V Congreso Nacional de Medicina. (1935), Tomo VIII. Rosario: Talleres gráficos Pomponio, pp. 281-287, pp. 399-408, pp. 309-312, pp. 351-353, pp. 374-378.

\section{BIBLIOGRAFÍA}

Allevi, José Ignacio (2016), “La profilaxis de la locura en la agenda política: saberes y técnicos de la Higiene Mental en la metamorfosis del Estado santafesino de entreguerras", Estudios Sociales del Estado, 2 (3), pp. 65-98.

Allevi, José Ignacio (2017), Sociabilidades, redes y expertos. La emergencia de un espacio de ciencia y clínica psi en la ciudad de Rosario (1920-1943), Universidad Nacional de La Plata, Tesis de Maestría.
37. Foz llegó a dirigir más de 80. Biblioteca de la Cátedra Psiquiatría de Adultos. Facultad de Ciencias Médicas. Universidad Nacional de Rosario.

38. Como fue el caso de Inocencio Ansaldi, Carlos Crespo, Pedro Nisibar, Nicolas Vita, Zoe Bula, Enrique Dallari y Manuel Pazo.

39. En torno a dicha institución cultural y de lucha antifascista, véase Celentano (2006).

40. "Informe elevado al Señor Decano de la Facultad de Ciencias Médicas, por el Señor Director del Instituto de Psiquiatría (1937)". Boletín del Instituto Psiquiátrico (1937), Año I, 2da época (20-21), p. 205.

41. No olvidemos aquí que Ciampi compartía vínculos no sólo con la $L A H M$, sino también con la Asociación Argentina de Biotipología, Eugenesia y Medicina Social o la Sociedad Argentina de Venerología y Profilaxis Social, ambas con orientaciones eugenésicas de corte fascista (Vallejo y Miranda, 2005).

42. Foz, Antonio (1938), "Eugenesia”. Boletín del Instituto Psiquiátrico, Año I, 2da época (23), pp.116-130.

43. Sebastián Soler fue un abogado especializado en Derecho Penal, recibido en la Universidad de Córdoba en 1924. En 1933 es designado camarista en lo Criminal en Rosario y desde 1934 fue profesor de Derecho Penal en la Universidad Nacional de Córdoba. Entre 1955 y 1958 se desempeñó como Procurador General de la Nación, y desde 1951 será miembro consultivo de la Secretaría General de la ONU en el cuerpo internacional de expertos en protección del crimen.

44. "Sociedad de Psiquiatría, Psicopatología y Medicina Legal". Boletín del Instituto Psiquiátrico (1937), Año I, 2da época (20-21), pp. 207-211.

45. "Hospital Nacional de Alienados. Memoria correspondiente al año 1938". Boletín del Instituto Psiquiátrico (1938), Año II, 2da época, (23), p. 226.

46. “Hospital Nacional de Alienados de la Facultad de Ciencias Médicas de Rosario. Memoria correspondiente al año 1939". Boletín del Instituto Psiquiátrico (1939), Año III, 2da época, (25), p.156; “Hospital Nacional de Alienados de la Facultad de Ciencias Médicas de Rosario. Memoria correspondiente al año 1940". Boletín del Instituto Psiquiátrico (1941), Año V, 2da época, (27), pp. 65-66.

Ameghino, Arturo (1929a), Actas de la Primera Conferencia Latino Americana de Neurología, Psiquiatría y Medicina Legal. Buenos Aires, Imprenta de la Universidad, Tomo I.

Ameghino, Arturo (1929b), Actas de la Primera Conferencia Latino Americana de Neurología, Psiquiatría y Medicina Legal. Buenos Aires, Imprenta de la Universidad, Tomo II.

Armus, Diego (2007), La ciudad Impura, Buenos Aires, Edhasa. 
Bidart, Claire y Cacciuttollo, Patrice (2009), “En busca del contenido de las relaciones sociales: los 'motivos' de las relaciones", REDES. Revista hispana para el análisis de redes sociales, vol. 6, (2), pp. 178-202.

Bosch, Raimundo (1966), Historia de la Facultad de Medicina, Rosario, Universidad del Litoral

Buchbinder, Pablo (2005), Historia de las universidades argentinas, Buenos Aires, Editorial Sudamericana.

Buchbinder, Pablo (2008), ¿Revolución en los claustros? La Reforma Universitaria de 1918, Editorial Sudamericana, Buenos Aires.

Buschini, José (2013), "Renovación institucional y modernización científica: la creación del Instituto de Investigaciones Hematológicas a mediados de la década de 1950", Salud Colectiva, 9 (3), pp. 317-334.

Carbonetti, Adrián (2003), "La formación de la tisiología como especialidad médica en Córdoba (Argentina), 1920-1950", Horizontes. Braganza paulista, 21, pp. 105-116.

Celentano, Adrián (2006), “Ideas e intelectuales en la formación de una red sudamericana antifascista", Literatura y Lingüística, 17, pp. 195-218, Santiago.

Dosse, Francois (2006), La marcha de las ideas. Historia de los intelectuales, historia intelectual, Valencia, PUV.

Farías, Ruy (2013), "El exilio de los intelectuales y científicos republicanos españoles y el mundo académico argentino: mediadores institucionales y trayectorias individuales", Revista UNIABEU, 6 (12), pp. 81-96.

Fernández, Sandra (2014), “Entre el orden científico y la beneficencia. La experiencia del Hospital e Instituto de Enseñanza Médica del Centenario. Rosario, 1910-1929". En: Bravo, María Celia y Fernández Sandra, Formando el espacio público: asociacionismos y política. Siglos, XIX y XX, EDUNT, Tucumán, p. $79-100$.

Ferrari, Fernando (2016), De la locura a la enfermedad mental. Córdoba, 1758-1930. Una historia cultural sobre los discursos y prácticas médicas sobre la locura, Córdoba.

García, Luciano (2012), “La recepción de la psicología soviética en la Argentina: lecturas y apropiaciones en la psicología, psiquiatría y psicoanálisis (1936-1991)", Facultad de Filosofía y Letras, UBA, Tesis Doctoral.

García, Luciano (2016), La psicología por asalto. Psiquiatría y cultura científica en el comunismo argentino (1935-1991), Buenos Aires, Edhasa.

Gentile, Antonio (2003), Ensayos históricos sobre psicoanálisis y psicología en la Argentina, Rosario, Fundación Ross.

Golcman, Alejandra (2015), "El diagnóstico de la demencia precoz y la esquizofrenia en Argentina, 1920-1940", Trashumante. Revista Americana de Historia Social, 5, pp. 150-172.

Golcman, Alejandra (2017), "Legitimar psiquiatras antes que curar pacientes. Las terapias de shock en Buenos Aires, Argentina (1930-1970)". Asclepio. Revista de Historia de la Medicina y de la Ciencia, vol. 69, (1), p176.
González Leandri, Ricardo (1999), Curar, persuadir, gobernar. La construcción histórica de la profesión médica en Buenos Aires, 1852-1886, Madrid, CSIC.

González Leandri, Ricardo (2012), "Itinerarios de la profesión médica y sus saberes de Estado en Buenos Aires, 18501910". En: Plotkin, Mariano; Zimmermann, Eduardo (eds.), Los saberes del Estado, Buenos Aires, Edhasa.

Grossetti, Michel (2009), “¿Qué es una relación social? Un conjunto de mediaciones diádicas. REDES. Revista hispana para el análisis de redes sociales, 6 (2), pp. 46-62.

Hess, Volker y Majeurs, Benoit (2011), "Writing the history of psychiatry in the 20th century", History of Psychiatry, vol. 22, (2), pp. 139-145.

Huertas, Rafael (2001), "Historia de la Psiquiatría ¿por qué? ¿para qué?. Tradiciones historiográficas y nuevas tendencias", Frenia, 1 (1), pp. 9-36.

Huertas, Rafael (2002), Organizar y persuadir. Estrategias profesionales y retóricas de la legitimación de la medicina mental española (1875-1936), Madrid, Frenia.

Huertas, Rafael (2008) Los laboratorios de la norma. Medicina y regulación social en el Estado liberal, Madrid, Octaedro-CSIC.

Huertas, Rafael (2012), Historia cultural de la psiquiatría. (Re) Pensar la locura, Madrid, Libros de la Catarata.

Kurowski, Maristela (2001), La obra psicológica de Juan Cuatrecasas Arumí (1899-1990). Tesis doctoral, Madrid, Universidad Complutense de Madrid.

Liscia, Ma. Silvia di (2003), Saberes, Terapias y Prácticas Médicas en Argentina (1750-1910), Biblioteca de Historia de América, Madrid, Consejo Superior de Investigaciones Científicas.

Marco, Miguel Ángel de (2015), "Universitarios rosarinos en la recepción de exiliados republicanos españoles. El decanato de Cortés Plá. 1934-1943", Épocas. Revista de Historia, 11, p. 41-70.

Meduna, Ladislao y Foz, Antonio (1938), “El tratamiento convulsivante de algunas enfermedades mentales". Boletín del Instituto Psiquiátrico, II (23), p. 168-182.

Miranda, Marisa (2013), "La Argentina en el escenario eugénico internacional". En: Miranda, Marisa y Vallejo, Gustavo, (eds.), Una historia de la eugenesia. Argentina y las redes biopolíticas internacionales 1912-1945, Buenos Aires, Biblos.

Pacheco, Pablo A. (2010), "El Centro de Estudio Físico Matemáticos de la Universidad Nacional de Cuyo (1948-1950)", Redes, 16 (31), pp. 199-214.

Pascual, Cecilia (2017), "La epidemia de cólera como condensador de sentidos. Culturas urbanas, narraciones clínicas y políticas higiénicas en Rosario, 1886/7", História, Ciências, Saúde-Manguinhos, 24 (2), pp. 295-311.

Perón-Magnan, Pierre (2000), "La era moderna de las terapéuticas biológicas". En: Postel, Jacques y Quétel, Claude, Nueva historia de la Psiquiatría, México, Fondo de Cultura Económica. 
Prieto, Agustina (1996), "Rosario: epidemias, higiene e higienistas en la segunda mitad del siglos XIX". En: Lobato, Mirta (ed.), Política, médicos y enfermedades. Lecturas de la historia de la salud en la Argentina, Buenos Aires, Biblos, pp. 57-71.

Ramacciotti, Karina y Valobra, Adriana (2010), "La profesionalización de la enfermería en Argentina: disputas políticas e institucionales durante el peronismo". Asclepio. Revista de historia de la medicina y de la ciencia, LXII (2), pp. 353-374.

Reggiani, Andrés (2016), "Cultura física, performance atlética e higiene de la nación. El surgimiento de la medicina dportiva en Argetina (1930-1940)", Historia Crítica, (61), pp. 65-84.

Rieznik, Marina (2009), “Debates en los observatorios argentinos. La creación de la Escuela Superior de Ciencias Astronómicas y Conexas (1935)", Redes, 15 (30), pp.19-40.

Romero, Lucía (2016), Entre pipetas, bisturíes y pacientes. La investigación clínica en la Argentina: la tradición Lanari. Buenos Aires, Biblos.
Talak, Ana María (2010). “Progreso, degeneración y darwinismo en la primera psicología argentina, 1900-1920". En Vallejo, G. y Miranda, M. (dirs.), Derivas de Darwin. Cultura y Política en clave biológica, Buenos Aires, Siglo XXI Iberoamericana.

Talak, Ana María (2008). La invención de una ciencia primera. Los primeros desarrollos de la psicología en la Argentina (18961919, Tesis doctoral, Facultad de Filosofía y Letras, UBA.

Talak, Ana (2005), “Eugenesia e higiene mental: usos de la psicología en la Argentina (1900-1940)". En: Miranda, Marisa y Vallejo, Gustavo (eds.), Darwinismo social y eugenesia en el mundo latino, Buenos Aires, Siglo XXI Iberoamericana.

Tarcus, Horacio (dir.) (2007), Diccionario biográfico de la izquierda argentina, Buenos Aires, Emecé Editores.

Vallejo, Gustavo y Miranda, Marisa (2005), "La eugenesia y sus espacios institucionales en la Argentina". En Miranda, Marisa; Vallejo, Gustavo (eds.), Darwinismo social y eugenesia en el mundo latino, Buenos Aires, Siglo XXI Iberoamericana.

Vezzetti, Hugo (1983), La locura en la Argentina, Buenos Aires, Editorial Folios. 